Check for updates

Cite this: Phys. Chem. Chem. Phys., 2020, 22, 1640

Received 15th November 2019 Accepted 16th December 2019

DOI: $10.1039 / c 9 c p 06199 a$

rsc.li/pccp

\section{Local anisotropy in single crystals of zeotypes with the MFI framework structure evidenced by polarised Raman spectroscopy $\dagger$}

\author{
Anna Martinelli, (D) *a Simone Creci, (D) ab Szilvia Vavra, (iD ${ }^{a}$ Per-Anders Carlsson (iD) ab \\ and Magnus Skoglundh iD ab
}

\begin{abstract}
Polarised Raman spectroscopy is used to characterise the local structure in single crystals of zeotypes, namely silicalite-1 and ZSM-5, which share the MFI framework structure. Attributes favourable for applying polarised Raman spectroscopy are the orthogonal axes of these single crystals and their size, i.e. 10 to 30 micrometers in all three directions. We show that the intensity of certain vibrational modes in silicalite-1 depends on the polarisation of the incident light, reflecting the anisotropic character of the molecular bonds contributing to these vibrations. Using these observations, and by estimating the depolarisation ratio $(\rho)$ and the pseudo-order factor $(f)$, we propose a more accurate assignment of the Raman active modes. More precisely, Raman intensities peaked at 294, 360, 383 and $472 \mathrm{~cm}^{-1}$ are attributed to bending modes in 10-, 6-, 5- and 4-membered rings, respectively. In the region of stretching modes, the vibration at $832 \mathrm{~cm}^{-1}$ is assigned to $\mathrm{Si}-\mathrm{O}-\mathrm{Si}$ bonds shared between 5 -membered rings, which have an orientation parallel to the a-axis of the crystal. By virtue of having a strongly polarised character, the modes at 472 and $832 \mathrm{~cm}^{-1}$ can be used as orientational indicators. The proposed assignment is supported by the good agreement between experimental and simulated polar plots, where Raman intensities are plotted as a function of the polarisation angle of the incident light. Finally, upon partial substitution of $\mathrm{Si}$ atoms by $\mathrm{Al}$, the crystalline structure is maintained and almost no spectroscopic changes are observed. The only significant difference is the increased width of most vibrational modes, which is consistent with the local lower symmetry. This is also seen in the angular dependence of selected vibrational modes that compared to the case of pure silicalite-1 appear less polarised. In the Raman spectrum of ZSM-5 a new feature at $974 \mathrm{~cm}^{-1}$ is observed, which we attribute to $\mathrm{Al}-\mathrm{OH}$ stretching. In the high frequency range, the $\mathrm{O}-\mathrm{H}$ stretching modes are observed which arise from the $\mathrm{Si}-\mathrm{O}(\mathrm{H})-\mathrm{Al}$ Brønsted acid sites. The intensity of the characteristic mode at $3611 \mathrm{~cm}^{-1}$ reveals an anisotropic character as well, which is in line with previous findings from solid state NMR that Al atoms distribute nonrandomly within the MFI framework structure.
\end{abstract}

\section{Introduction}

Zeolites are materials of major scientific and technological importance. They have a huge commercial value and are used in a wide range of fields such as adsorption processes, ion exchange, gas separation, and catalysis. The chemical structure of zeolites includes $\mathrm{Si}, \mathrm{Al}$, and $\mathrm{O}$ atoms, ordered in different

\footnotetext{
${ }^{a}$ Department of Chemistry and Chemical Engineering, Chalmers University of Technology, SE-412 96 Gothenburg, Sweden. E-mail: anna.martinelli@chalmers.se

${ }^{b}$ Competence Centre for Catalysis, Chalmers University of Technology, SE-412 96 Gothenburg, Sweden

$\dagger$ Electronic supplementary information (ESI) available: Additional Raman spectra, peak-fit models used to extract Raman intensities, complete set of Raman tensor coefficients, additional polar plots, polar plots for the ZSM-5. See DOI: 10.1039/ с9ср06199a
}

ways depending on the adopted crystalline structure, while counter-ions, typically $\mathrm{H}^{+}$or $\mathrm{NH}_{4}{ }^{+}$, balance the net negative charges caused by the presence of aluminium atoms in the framework structure. In addition, zeolites are microporous materials with high surface area and high degree of crystallinity, with characteristic crystal structures that by convention are described by a 3-letter code (e.g. MFI, CHA, etc.). If $\mathrm{Al}$ is not present in the structure, or if this is substituted for another element, the material is referred to as a zeotype.

The hierarchical and crystalline structure of zeolites has been studied by high resolution methods like TEM and XRD, ${ }^{1-3}$ while the challenging question related to the position and distribution of $\mathrm{Al}$ atoms in the framework structure has been addressed by NMR and DFT methods. ${ }^{4-6}$ High-quality TEM images of zeolites require advanced sample preparation, while 
both XRD and solid state NMR methods are applied to samples in powder form whereby the local orientation characteristic of single crystals cannot be probed. Infrared spectroscopy is an established method for quantitative studies of chemical reactions occurring inside the pores of zeolites, but vibrational spectroscopy in general (including Raman spectroscopy, infrared spectroscopy and inelastic neutron scattering) has been sparingly used to characterise the framework structure in zeolites. ${ }^{7-16}$ Infrared spectroscopy does not provide sufficiently high spectral resolution to clearly distinguish different zeolite structures through their characteristic vibrational modes, ${ }^{9,11}$ while the use of Raman spectroscopy has been limited due to problems of luminescence (in particular in zeolites containing Fe atoms), a low scattering cross section, and many times minor spectroscopic changes upon atomic substitution $($ e.g. $\mathrm{Si} \rightarrow \mathrm{Al}) .^{7-9,13}$ Inelastic neutron scattering has the disadvantage of difficult access to neutron facilities. As a result of these aspects, infrared spectroscopy has been used about ten times more than Raman spectroscopy, and hundred times more than neutron scattering. ${ }^{17}$ To compensate for these experimental limitations, complementary theoretical calculations have been performed with the aim to elucidate distinct spectral features arising from specific and isolated structural sub-units (such as double 4-membered rings or double 6-membered rings, etc.). ${ }^{10,12,14,15,18}$ Group theory has also been invoked to break down the complexity in vibrational spectra of zeolites, which although representing an important contribution also showed that many zeolites of technological and scientific importance are in fact poor candidates for fulllattice dynamics analysis due to the large number of atoms in the Bravais unit cell, and the low space group and site group symmetry. ${ }^{7}$ Maroni anticipated already in $1988^{7,19}$ the potential usefulness of polarised Raman spectroscopy on single crystals of zeolites for an otherwise impossible peak assignment. Since then this method has been used to study zeolites, albeit mainly for the detection and orientation of molecular species hosted inside the pores $^{20,21,23,24}$ but less frequently with the specific aim to assign intrinsic vibrational modes using single crystals. ${ }^{20,22}$ In a broader context, and based on its sensitivity to crystals' anisotropy, polarised Raman spectroscopy has been used in structural studies of biological systems, ${ }^{25}$ in several studies of stretched polymer fibres, ${ }^{26,27}$ to define the orientation of molecular species hosted in micro- and nano-pores, ${ }^{28}$ and to assign vibrational modes in crystalline materials. ${ }^{18,29-32,34}$

In the present work, we demonstrate that polarised Raman spectroscopy is sensitive also to the anisotropies of single crystals of zeolites and that, as a consequence, distinction between vibrational modes can be made based on the orientation of certain chemical bonds. More precisely we apply polarised Raman spectroscopy to single crystals of silicalite-1 and ZSM- 5 that both share the MFI framework structure, see Fig. 1. Despite the complex multiple-ring structure, the MFI framework has crystal axes orthogonal to each other and hence parallel to the laboratory coordinate system, which facilitates the interpretation of the collected Raman spectra. Polarised and angular dependent Raman intensities are presented and discussed, and used to make an assignment of the observed vibrational modes more precise
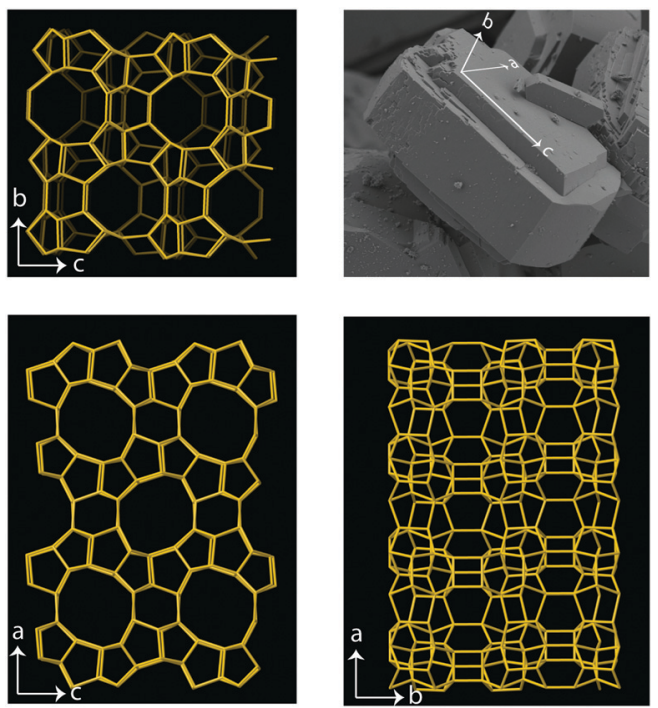

Fig. 1 Top-right: SEM image showing the characteristic shape of a single zeolite crystal with the MFI framework structure, the crystal axes are also illustrated. The length of this crystal along the $c$-axis is about $35 \mu \mathrm{m}$. The local order of a zeotype with the MFI framework structure is given for the $b-c$ plane (top-left), the $a-c$ plane (bottom-left), and the $a-b$ plane (bottom-right) to reveal the complex combination of 4-, 5-, 6- and 10 -membered rings that build up the crystal. The large pores (i.e. the 10-membered rings) are aligned along the $b$-direction whereas they are oriented zig-zag along the a-direction.

than is available today. The study of Al-free, i.e. purely siliceous, crystals is intentionally included to evidence the correlation between framework structure and vibrational modes, and show that the majority of frequencies and intensities are maintained upon substitution of $\mathrm{Si}$ with $\mathrm{Al}$ atoms. With this insight we provide pivotal clarifications on the origin of vibrational modes in zeolites of the MFI structure, which have some times been erroneously assigned. For example, vibrational modes in the Raman spectrum at 438, 745, 814 and $832 \mathrm{~cm}^{-1}$ have been assigned to $\mathrm{Si}-\mathrm{O}-\mathrm{Al}$ stretching and $\mathrm{O}-\mathrm{Si}-\mathrm{O}(\mathrm{Al})$ bending modes ${ }^{33}$ although these, as shown in this work, are observed at exactly the same frequencies and intensities in the Al-free structure. This paper is intended to be a source of inspiration for further theoretical and experimental studies, but also to promote a new useful method to identify the site of metal atoms substituting for $\mathrm{Si}$, a crucial aspect in catalytic materials.

\section{Experimental section}

\subsection{Synthesis and characterisation}

Samples of silicalite- 1 and ZSM-5 with the MFI type of framework structure were prepared according to the method proposed by Szotak et $a .^{35}$ The chemicals used for the synthesis were the following: tetraethyl orthosilicate (TEOS, reagent grade, 98\%, Sigma Aldrich) as silicon source, sodium aluminate (Technical, anhydrous, Sigma Aldrich) as aluminum source and tetrapropylammonium hydroxide (TPAOH, 1.0 M Lsg. in water, SigmaAldrich) as structure directing agent (SDA). Oxalic acid (98\%, Sigma-Aldrich) and sodium hydroxide (pellets, 98\%, Alfa Aesar) 
were used to adjust the $\mathrm{pH}$ value. The molar ratio $\mathrm{Si}: \mathrm{TPA}^{+}: \mathrm{H}_{2} \mathrm{O}=$ $1.00: 0.30: 24.86$ was kept constant in both syntheses. For the ZSM-5 samples, TEOS was first hydrolysed for $24 \mathrm{~h}$ at $60{ }^{\circ} \mathrm{C}$ in water and oxalic acid ( $\mathrm{pH} 2$ ). The $\mathrm{Al}$ precursor was dissolved in water for $5 \mathrm{~h}$ (for the ZSM-5 sample, two solutions were prepared) and added to the TEOS containing solution. The solution was kept at $\mathrm{pH} 2$ in order to prevent precipitation of metal hydroxide species. After $4 \mathrm{~h}$ of stirring, a $20 \mathrm{wt} \%$ solution of TPAOH was added dropwise under vigorous stirring and then the $\mathrm{pH}$ was adjusted with a $1 \mathrm{M} \mathrm{NaOH}$ solution to the basic conditions necessary to promote crystallisation ( $\mathrm{pH} 11)$. The gel was heated in an unstirred autoclave (0.2 L, Parr Instrument Company) for 5 days at $170{ }^{\circ} \mathrm{C}$. The obtained powder was washed, filtered and instantaneously frozen with liquid nitrogen and freeze-dried overnight. In order to remove the SDA and to obtain the $\mathrm{Na}^{+}$-form of the zeolite, the as synthesised sample was calcined at $500{ }^{\circ} \mathrm{C}$ for $5 \mathrm{~h}$ in air $\left(5^{\circ} \mathrm{C} \mathrm{min}^{-1}\right)$. The $\mathrm{Na}^{+}$-zeolite sample was then ionexchanged twice at $60{ }^{\circ} \mathrm{C}$ for $24 \mathrm{~h}$ with ammonium nitrate (EMPLURA, Sigma-Aldrich), followed by washing, filtration and freeze-drying overnight. The $\mathrm{H}^{+}$-form of the zeolite was obtained by calcination at $500{ }^{\circ} \mathrm{C}$ for $1 \mathrm{~h}$ in air $\left(10{ }^{\circ} \mathrm{C} \mathrm{min}{ }^{-1}\right)$. The ZSM-5 samples was prepared with a $\mathrm{Si}: \mathrm{Al}$ molar ratio (SAR) equal to 50 . For the silicalite-1 samples, the same procedure as for ZSM-5 was followed, but no Al precursor was used and the ammonium exchange and the second calcination were not performed.

The morphology on the micrometer scale of the calcined crystals of silicalite- 1 and ZSM- 5 was investigated by scanning electron microscopy (SEM), using a Zeiss Ultra 55 FEG instrument. The acceleration voltage was set to $1.5 \mathrm{kV}$ and the samples were imaged without any coating. The X-ray diffractograms of the calcined samples of silicalite-1 and ZSM-5 were recorded with a Bruker XRD D8 advanced instrument with monochromatic $\mathrm{Cu} \mathrm{K} \alpha$ radiation, scanning from $5^{\circ}$ to $55^{\circ}$ with a step size of $0.029^{\circ}$ and a dwell time of $1 \mathrm{~s}$. In the case of silicalite-1, the diffractogram shows the characteristic peaks congruent with the MFI framework structure already reported for crystals prepared as in this work ${ }^{36}$ and a high degree of crystallinity, Fig. 2a. The corresponding SEM image shows distinct single crystals, homogeneous in size and shape, that have an average length of about 20 micrometers along the $c$-axis and of 12 micrometers along the $a$-axis. The height of the crystals along the $b$-axis is on average 10 micrometers. The XRD pattern recorded from samples of ZSM-5 is equivalent to that of silicalite-1, confirming the common MFI framework structure, Fig. 2b. The morphology of ZSM-5 single crystals is also the same, although the crystals are on average larger in size. An important note is that for both pure silicalite- 1 and ZSM- 5 no morphological changes are observed between before and after calcination.

\subsection{Polarised Raman spectroscopy}

Raman spectra were recorded with an InVia Reflex spectrometer purchased from Renishaw. A high power near-infrared diode laser with a maximum output power of $300 \mathrm{~mW}$ and a wavelength of $785 \mathrm{~nm}$ was used as the excitation source. This, together with a grating with 1200 lines per $\mathrm{mm}$ allowed achieving a

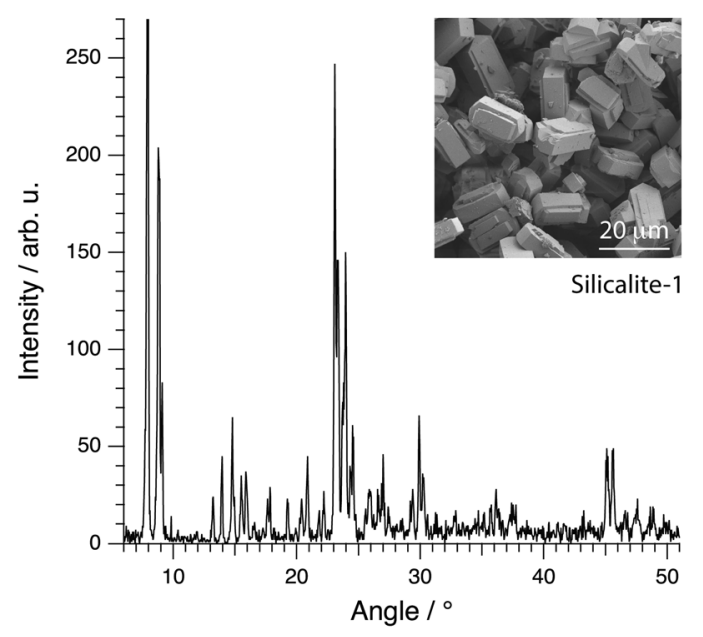

a)

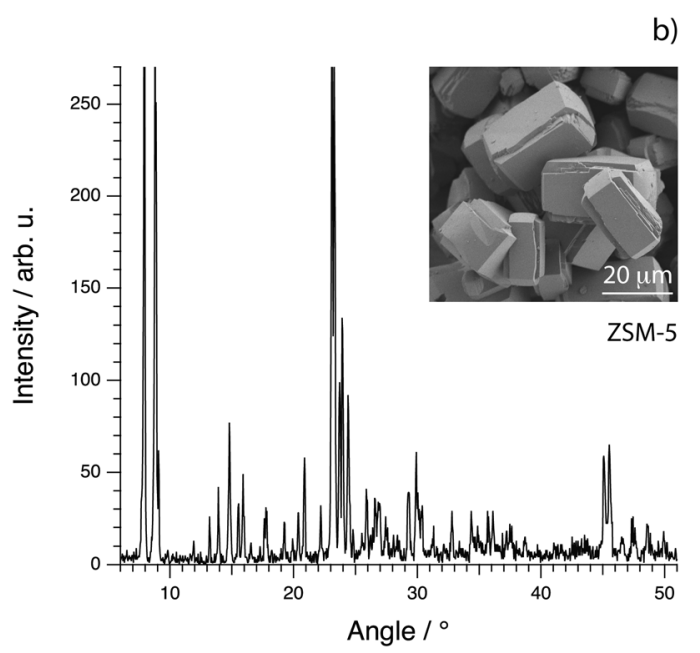

Fig. 2 X-ray diffractograms (main figures) and SEM images (insets) for the synthesised crystals of silicalite-1 (a) and ZSM-5 (b).

spectral resolution of about $1 \mathrm{~cm}^{-1}$. A motorised polarisation kit along with an analyser that is possible to set in the vertical or horizontal mode were coupled and used to collect polarisation sensitive Raman spectra. In this work, and due to the technical specifications of the spectrometer used, normally polarised light corresponds to polarisation along the $x$-axis of the laboratory coordinates, while orthogonally polarised light corresponds to polarisation along the $y$-axis. This, and other configurational details are illustrated in Fig. 3. In order to collect Raman spectra at variable polarisation angles, the polarisation of the incident light, rather than the single crystal, was rotated. This deliberate choice is crucial to ensure that the same volume of material is illuminated (and hence investigated) and to avoid intensity changes due to misalignment or local density fluctuations. All spectra were collected in the backscattering mode $\left(180^{\circ}\right)$ with the direction of the incident and scattered light being parallel to the $z$-axis. A Leica objective with numerical aperture (NA) 0.50 and magnification $50 \times$ was used to focus the incident light on selected spots over the single crystal's upper surface, enabling confocal spots of approximately $1 \times 1 \mu \mathrm{m}$ on the $x-y$ plane (this has been estimated using the relation 


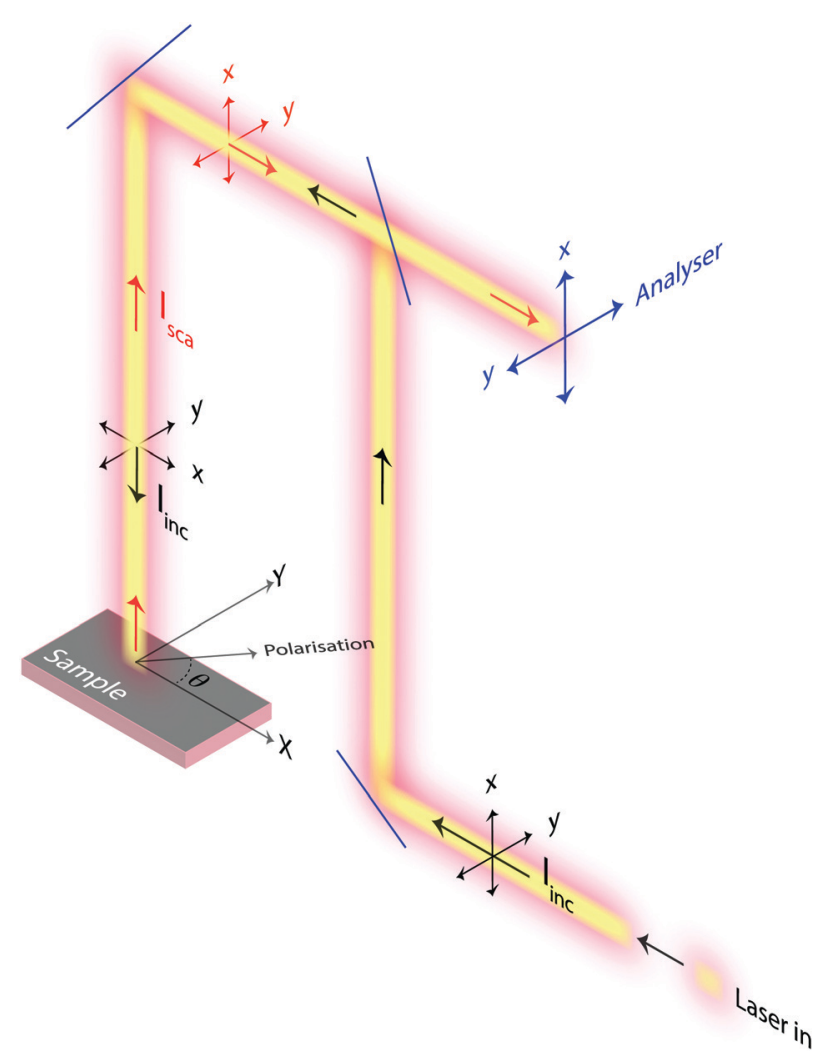

Fig. 3 Schematic of the experimental set up used in this study, showing the paths of the incident light $\left(l_{\text {inc }}\right.$, black arrows) and of the back scattered light $\left(I_{\mathrm{sca}}\right.$, red arrows). For both, the direction of the two possible polarisations are indicated. At the sample point, the direction of propagation for $I_{\text {inc }}$ and $I_{\text {sca }}$ is along the $\vec{z}$ axis, while the polarisation of light is along directions parallel to the $x-y$ plane which can also be described by $\theta$. Before the detector, an analyser can be placed to select either vertically (parallel to $x$ ) or orthogonally (parallel to $y$ ) polarised back scattered light.

$d=1.22 \cdot \lambda / \mathrm{NA}$, where $d$ is the diameter of the focal spot, $\lambda$ is the wavelength of the incident laser, and NA is the numerical aperture of the objective). Out of a number of single crystals randomly spread out over a foil of aluminium, those with the right orientation with respect to the laboratory axes were searched for using a low magnification objective and selected for further investigation. Crystal twins grown into each other or a crystal laying over another should not be selected. Spectra were then recorded collecting over 60 seconds and 3 accumulations to obtain a high signal-to-noise ratio for reliable analyses of Raman intensities. In this paper, and if not stated otherwise, with Raman intensity we intend the integrated area under a peak. Moreover, when peak fitting is applied a linear background and Lorentzian functions are used to fit the experimentally recorded spectra.

\section{Theoretical aspects}

The basic principles of Raman scattering are thoroughly described in established text books and review articles. Here, however, we recall some aspects that are crucial for the purpose of this specific work, in particular the angle dependence of Raman intensities.
Frequency dependent Raman intensities can be recorded due to an interaction between the incident light (typically in the visible range) and matter, which induces a dipole moment $(\mu)$ in the molecule or group of molecules investigated. This oscillating dipole moment emits light with a frequency that is modulated by that of the incoming light and an energy that is equal, higher or lower than that of the incident light. The relevant relation for a certain molecular vibration is thus:

$$
\begin{aligned}
\mu= & \alpha \cdot E=\alpha_{0} \cdot E_{0} \cdot \cos \left(2 \pi \nu_{0} t\right) \\
& +\frac{1}{2}\left(\frac{\partial \alpha}{\partial q}\right) q \cdot E_{0} \cdot \cos \left(2 \pi \nu_{0}-\nu_{\mathrm{m}} t\right) \\
& +\frac{1}{2}\left(\frac{\partial \alpha}{\partial q}\right) q \cdot E_{0} \cdot \cos \left(2 \pi \nu_{0}+\nu_{\mathrm{m}} t\right)+\ldots
\end{aligned}
$$

where $\alpha_{0}$ is the polarisability of the molecular bond at equilibrium, $E_{0}$ is the amplitude of the electric field in the incident light, $\nu_{0}$ is the frequency of the incident light, $t$ is time, $q$ is the displacement of the atom involved in the vibration from the equilibrium position, and $\nu_{\mathrm{m}}$ is the frequency of the vibration. In this equation, the first term represents Rayleigh scattering (i.e. light scattered with the same energy as the incident light) while the second and third terms represent Stokes and antiStokes Raman scattering, respectively. The condition for a vibrational mode to be Raman active is thus that $\left(\frac{\partial \alpha}{\partial q}\right) \neq 0$ at equilibrium position, which means that the molecular polarisability of the vibrational mode should be neither maximum nor minimum at $q=0$. With the spectrometer used in this work only light scattered with a lower energy than the incident light is detected, that is only the Stokes Raman events are measured. In general, the recorded Raman intensity is proportional to the square of the induced dipole moment but is also dependent on the power of the incoming light and the number of scatterers present within the focal volume of the incident laser. Moreover, when using polarised Raman spectroscopy it is crucial to realise that the induced dipole moment $\vec{\mu}$ is a vector with components in all directions $(x, y$, and $z)$ that depend on the electric field $\vec{E}$ through the polarisability Raman tensor. Further, the vector $\vec{\mu}$ has components parallel and orthogonal to $\vec{E}$.

For the sake of simplicity, we consider the case of an incident light propagating along the $z$-direction and thus being polarisable at directions parallel to the $x-y$ plane, as shown in Fig. 3. From a sample at a fixed position, Raman spectra can be recorded by setting different experimental configurations, for example with the incident light linearly polarised along the $x$-axis, along the $y$-axis or by varying the direction of polarisation around the angle $\theta$. Further, by placing an analyser between the sample and the detector, scattered light with a polarisation parallel either to the $x$-axis or the $y$-axis can be selected to reach the detector. Without an analyser, scattered light with polarisation in all directions will be recorded. Mathematically, the Raman intensity will be proportional to $\left|\vec{e}_{\text {inc }} \cdot R \cdot \vec{e}_{\text {sca }}\right|^{2}$, where $\vec{e}_{\text {inc }}$ is the unit vector describing the direction of the incident light, $\vec{e}_{\text {sca }}$ is the unit vector describing the direction of the scattered 
light, and $R$ is the Raman polarisability tensor. In the specific case where $\vec{e}_{\text {sca }}$ is parallel to $\vec{e}_{\text {inc }}$ we can express the product $\vec{e}_{\text {inc }}$. $R \cdot \vec{e}_{\text {sca }}$ as:

$$
\left(\begin{array}{ll}
\cos \theta & \sin \theta
\end{array}\right)\left(\begin{array}{cc}
\alpha_{x x} & \alpha_{x y} \\
\alpha_{y x} & \alpha_{y y}
\end{array}\right)\left(\begin{array}{c}
\cos \theta \\
\sin \theta
\end{array}\right)
$$

which rearranges to

$$
\left(\begin{array}{ll}
\cos \theta & \sin \theta
\end{array}\right)\left(\begin{array}{c}
\alpha_{x x} \cos \theta+\alpha_{x y} \sin \theta \\
\alpha_{y x} \cos \theta+\alpha_{y y} \sin \theta
\end{array}\right)
$$

and then further to

$$
\left(\alpha_{x x} \cos ^{2} \theta+\alpha_{x y} \cos \theta \sin \theta+\alpha_{y x} \cos \theta \sin \theta+\alpha_{y y} \sin ^{2} \theta\right)
$$

The latter expression can be rewritten using trigonometric identities. If $\alpha_{y x}=\alpha_{x y}$, it becomes $\alpha_{x x} \cos ^{2} \theta+\alpha_{x y} \sin ^{2} \theta+\alpha_{y y} \sin ^{2} \theta$. From this it follows that the Raman intensity measured for a symmetric vibration at parallel polarisation (i.e. when $\vec{e}_{\text {sca }}$ is parallel to $\vec{e}_{\text {inc }}$ ) is expected to be:

$$
I_{\|}^{\mathrm{sS}} \propto\left(\alpha_{x x} \cos ^{2} \theta+\alpha_{y y} \sin ^{2} \theta\right)^{2}
$$

since $\alpha_{x y}$ and $\alpha_{y x}$ in the Raman tensor are considered to be negligibly small. Analogously, the Raman intensity recorded at parallel polarisation for an anti-symmetric vibration should be:

$$
I_{\|}^{\text {as }} \propto\left(\alpha_{x y} \sin ^{2} \theta\right)^{2}
$$

In the case of cross polarisation, i.e. when $\vec{e}_{\text {sca }}$ and $\vec{e}_{\text {inc }}$ are orthogonal to each other, the following product should be considered instead:

$$
\left(\begin{array}{ll}
\cos \theta & \sin \theta
\end{array}\right)\left(\begin{array}{ll}
\alpha_{x x} & \alpha_{x y} \\
\alpha_{y x} & \alpha_{y y}
\end{array}\right)\left(\begin{array}{c}
-\sin \theta \\
\cos \theta
\end{array}\right)
$$

which has the solution $\left(\alpha_{y y}-\alpha_{x x}\right) \cos \theta \sin \theta+\alpha_{x y}\left(\cos ^{2} \theta-\sin ^{2} \theta\right)$. As a consequence, the Raman intensity measured for a symmetric vibration at cross polarised conditions is:

$$
I_{\perp}^{\mathrm{ss}} \propto\left(\alpha_{y y}-\alpha_{x x}\right)^{2} \cos ^{2} \theta \sin ^{2} \theta
$$

while the Raman intensity of an anti-symmetric vibration will be associated to:

$$
I_{\perp}^{\text {as }} \propto\left(\alpha_{x y}\right)^{2}\left(\cos ^{2} \theta-\sin ^{2} \theta\right)^{2}
$$

If experiments are performed with the light polarised along the $x$-axis and only scattered light with a polarisation along the $x$-axis is allowed to reach the detector, both $\vec{e}_{\text {inc }}$ and $\vec{e}_{\text {inc }}$ can be described as the vector $(1,0)$ and the recorded Raman intensity will be proportional to $\left|\alpha_{x x}\right|^{2}$. This is equivalent to performing an experiment at parallel polarisations, and according to the Porto notation this experiment is described by the $z(x x) \bar{z}$ configuration. Similarly, if the incident light is polarized along the $y$-axis and only scattered light with a polarisation along the $y$-axis is detected, $\vec{e}_{\text {inc }}$ and $\vec{e}_{\text {sca }}$ can be described as the $(0,1)$ vector and the Raman intensity will be proportional to $\left|\alpha_{y y}\right|^{2}$. According to the Porto notation, this experiment is defined to have the $z(y y) \bar{z}$ configuration. If incident and scattered light are cross polarised, for example if $\vec{e}_{\text {inc }}$ is $(1,0)$ and $\vec{e}_{\text {sca }}$ is $(0,1)$, the
Table 1 Different possible experimental configurations specified by the polarisation of the incident $\left(\vec{e}_{\text {inc }}\right)$ and scattered $\left(\vec{e}_{\text {sca }}\right)$ light, the measured Raman intensity $\left(I_{i j}\right)$, the commonly used Porto notation, and the proportionality of the Raman intensity $\left(l^{R}\right)$ to the coefficients of the Raman tensor $\left(\alpha_{i j}\right)$

\begin{tabular}{lllll}
$\begin{array}{l}\text { Polarisation } \\
\text { of } \vec{e}_{\text {inc }}\end{array}$ & $\begin{array}{l}\text { Polarisation } \\
\text { of } \vec{e}_{\text {sca }}\end{array}$ & $\begin{array}{l}\text { Measured } \\
\text { intensity }\end{array}$ & $\begin{array}{l}\text { Porto } \\
\text { notation }\end{array}$ & $I^{\mathrm{R}}$ proportional to \\
\hline$\| x$ or $(1,0)$ & $\| x$ or $(1,0)$ & $I_{x x}$ & $z(x x) \bar{z}$ & $\left|\alpha_{x x}\right|^{2}$ \\
$\| x$ or $(1,0)$ & $\| y$ or $(0,1)$ & $I_{x y}$ & $z(x y) \bar{z}$ & $\left|\alpha_{x y}\right|^{2}$ \\
$\| y$ or $(0,1)$ & $\| y$ or $(0,1)$ & $I_{y y}$ & $z(y y) \bar{z}$ & $\left|\alpha_{y y}\right|^{2}$ \\
$\| y$ or $(0,1)$ & $\| x$ or $(1,0)$ & $I_{y x}$ & $z(y x) \bar{z}$ & $\left|\alpha_{y x}\right|^{2}$ \\
$(\cos \theta, \cos \theta)$ & $\| x$ or $(1,0)$ & $I_{\theta x}$ & - & $\left|\alpha_{x x} \cos \theta+\alpha_{y x} \sin \theta\right|^{2}$ \\
$\| y$ or $(0,1)$ & $(\cos \theta, \cos \theta)$ & $I_{y \theta}$ & - & $\left|\alpha_{y x} \cos \theta+\alpha_{y y} \sin \theta\right|^{2}$
\end{tabular}

solution of the $\vec{e}_{\text {inc }} \cdot R \cdot \vec{e}_{\text {sca }}$ product will be $\alpha_{x y}$, the recorded Raman intensity will be proportional to $\left|\alpha_{x y}\right|^{2}$, and the experiment is defined by the $z(x y) \bar{z}$ notation. The cases discussed represent well the role of the tensor's coefficients, since by their relative magnitude they determine at which angle intensity maxima are detected. In fact, diagonal values contribute to intensities recorded at parallel polarisations, while off-diagonal coefficients contribute to intensities recorded in cross polarised measurements. In this context, it is also worth mentioning that each vibrational mode is characterised by a unique Raman tensor. To summarise, a selection of different possible experimental configurations together with the associated Porto notation are given in Table 1. In Fig. 4, the simulated contributions from purely symmetric (ss) or anti-symmetric (as) vibrations when using a polarisation parallel $(\|)$ or orthogonal $(\perp)$ to the incident light are illustrated for fictitious values of $\alpha_{x x}, \alpha_{y y}, \alpha_{x y}$ and $\alpha_{y x}$ to clarify on their typical angular dependence.

In this work, we have specified the Cartesian coordinates as in Fig. 3, and assumed that these coincide with the coordinates
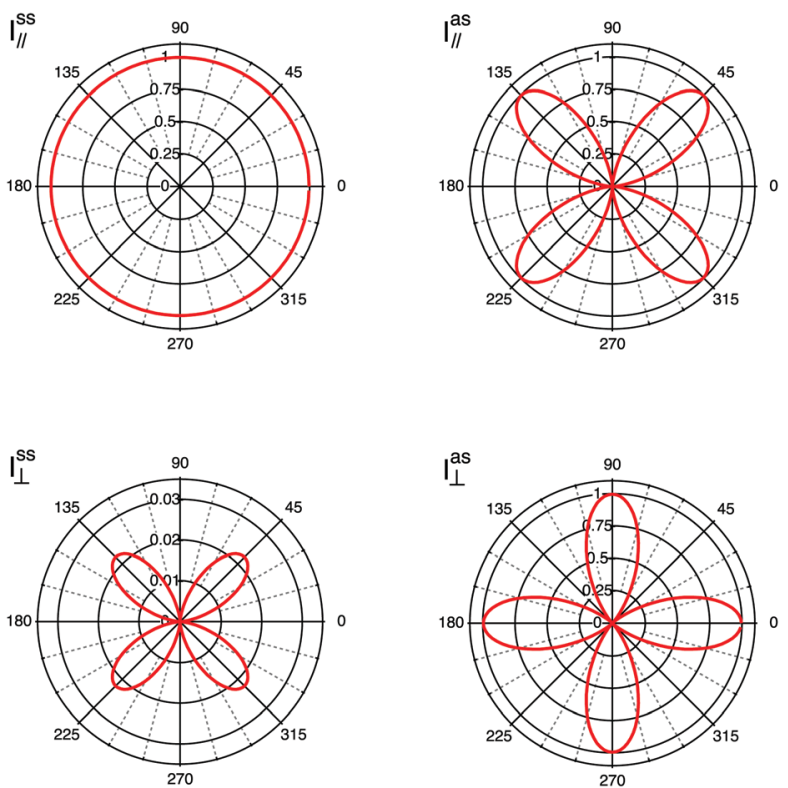

Fig. 4 Simulated polar plots considering purely symmetric (ss) and purely antisymmetric (as) modes contributing parallel (\|) or orthogonally ( $\perp$ ) to the incident light. The fictitious values of $\alpha$ are chosen as follows: $\alpha_{x x}=$ $\alpha_{y y}=1$ for the simulation of $I_{\|}^{\text {ss }} ; \alpha_{x y}=1$ for the simulation of $I_{\|}^{\text {as. }} ; \alpha_{x x}=0.7$ and $\alpha_{y y}=1$ for the simulation of $I_{\perp}^{\text {ss }}$; and $\alpha_{x y}=\alpha_{y x}=1$ for the simulation of $I_{\perp}^{\text {as }}$. 


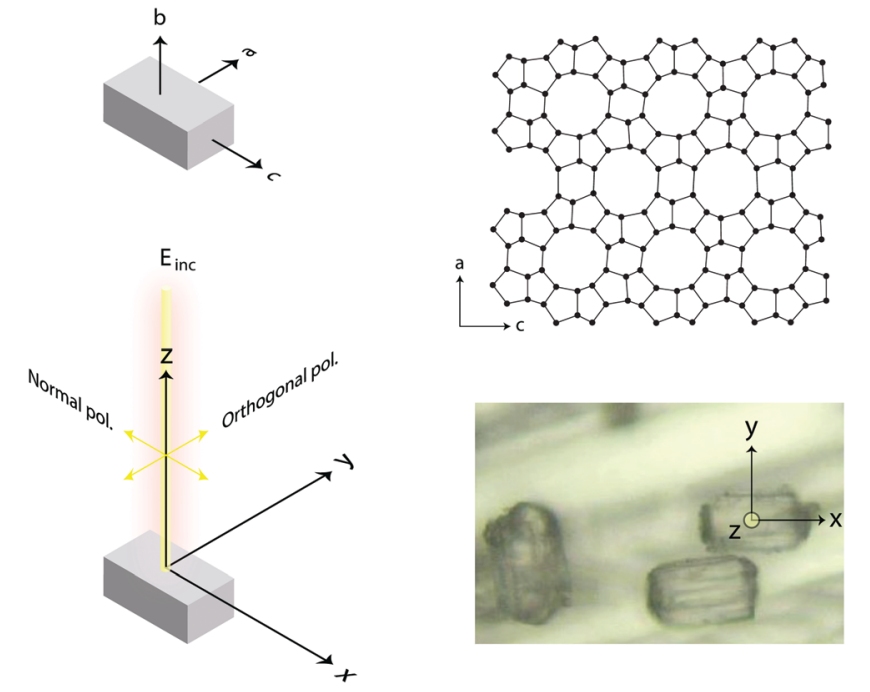

Fig. 5 Schematic view of the laboratory coordinates $x, y$, and $z$ (bottom left), of the crystal's axes $a, b$, and $c$ (top left), as well as the projection of the ring units building up the $a-c$ upper surface of the crystal (top right). The spot on the single crystal's surface from which the Raman spectra were collected is also precisely indicated (bottom right).

of the molecular system. This is not an obvious assumption in general but has been successfully used in many previous works. In the case of this study, however, we know from previous crystallographic investigations that single crystals of silicalite-1 do have axes of orientations that are orthogonal to each other and parallel to the crystal's faces. ${ }^{1}$ Most importantly, the MFI structure typical of this zeolite has also been confirmed by our X-ray results, see Fig. 2 and Section 2.1. This implies that the three axes in our laboratory coordinate system and the axes of the single crystals investigated are parallel, see also details in Fig. 5; hence no further angular transformations are needed for the interpretation of Raman intensities.

Based on the theory explained above, cross polarised measurements can be performed to extract values of the depolarisation ratio $(\rho)$ for a vibrational mode, where $\rho=I_{\perp} / I_{\|}$(in practice $\rho=I_{x y} / I_{x x}$ or $\left.\rho=I_{y x} / I_{y y}\right)$. By comparing the intensity detected at these different configurations it is possible to estimate the coefficients of the Raman tensor, which helps in assigning observed features to a specific vibrational mode. Complementary measurements done by variating the direction of polarisation of the incident light (that is the angle $\theta$, by either rotating the sample on the $x-y$ plane or rotating the polarisation of the incident light while keeping the sample fixed) serve to study the periodicity of Raman intensities, which are most appropriately shown in polar plots, as will be discussed in more details below.

\section{Results and discussion}

\subsection{Vibrational modes}

Raman spectra recorded at different polarisation configurations were recorded on single crystals of silicalite- 1 oriented to have the $c$-axis perfectly parallel to the $x$-axis, the $a$-axis parallel to the $y$-axis, and the $b$-axis parallel to the $z$-axis, as

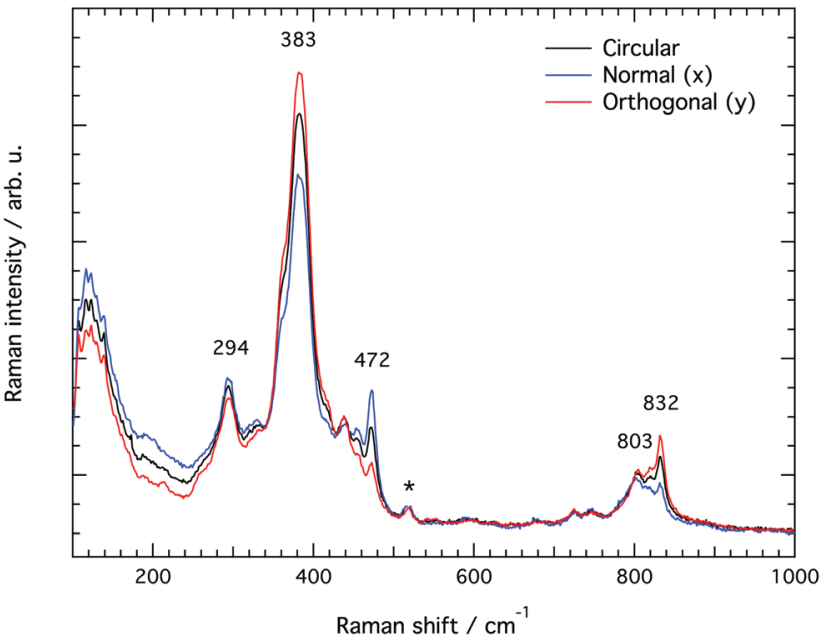

Fig. 6 Raman spectra collected from the upper $a-c$ plane of a single crystal of silicalite-1 with the incident light being circularly (black trace), normally (blue trace, parallel to the $x$-axis) and orthogonally (red trace, parallel to the $y$-axis) polarised. The asterisk marks a signature arising from the aluminium foil over which the single crystal is laying.

illustrated in Fig. 5. This figure also shows the ring structure that fills the space over the $a-c$ plane of the crystal, ${ }^{1}$ which includes 10-membered, 5-membered and 6-membered rings (see also Fig. 1). Tilted side views of this plane show that these rings are not perfectly planar (these and further structural details on this type of zeolite can be found in www.europe. iza-structure.org). Different planes along the $b$-direction are then connected by a complex combination of 4 -membered, 5-membered and 6-membered rings.

The Raman spectra recorded focusing the laser beam within 4 micrometers from the crystal's upper surface using circularly, normally (\| to $x$ ) and orthogonally (\| to $y$ ) polarised light are given in Fig. 6 . To note that these spectra were collected without an analyser before the detector to gain maximum intensity. In the frequency region below $600 \mathrm{~cm}^{-1}, \mathrm{Si}-\mathrm{O}-\mathrm{Si}$ bending modes are observed, while in the range $650-850 \mathrm{~cm}^{-1}$, modes assigned to $\mathrm{O}-\mathrm{Si}-\mathrm{O}$ and $\mathrm{Si}-\mathrm{O}-\mathrm{Si}$ stretching are detected. ${ }^{7-10,12-14,16,38}$ A more precise assignment of the observed Raman active modes, for instance one that is able to distinguish rings of specific size (e.g. 5-membered from 6-membered rings), has been very difficult for zeolitic materials due to energetically close vibrations and frequency shifts that depend on both the ring size, the bond angle, and the type of connection between the ring sub-units. To understand the complex correlation between local structure and vibrational modes, one can consider that by using the exact atomic position derived from a precedent refined crystallographic study, ${ }^{2}$ and the fact that silicalite- 1 has the orthorhombic symmetry and belongs to the space group Pnma (with 288 atoms per Bravais cell), the expected number of Raman active vibrational modes is as follows: $110 \mathrm{~A}_{\mathrm{g}}, 106 \mathrm{~B}_{1 \mathrm{~g}}, 110 \mathrm{~B}_{2 \mathrm{~g}}$ and $106 \mathrm{~B}_{3 \mathrm{~g}}{ }^{37}$

Nevertheless, based on precedent theoretical and experimental works, and on similarities to the local structure of vitreous silica, ${ }^{38-42}$ it is now established that the $\mathrm{Si}-\mathrm{O}-\mathrm{Si}$ stretching modes arise primarily from the movement of $\mathrm{Si}$ atoms with the $\mathrm{O}$ atoms 
considered fixed at their position, while the $\mathrm{Si}-\mathrm{O}-\mathrm{Si}$ bending, ring opening and deformation modes, are dominated by the displacement of $\mathrm{O}$ atoms along the $\mathrm{Si}-\mathrm{O}-\mathrm{Si}$ bisector in the plane of the structural unit, resulting in $\mathrm{Si}-\mathrm{O}-\mathrm{Si}$ bond angles that variate during the vibration. ${ }^{42}$ Moreover, the frequency of the O-bending modes is expected to red shift with increasing ring size, ${ }^{8,42}$ while the intensity (i.e. the Raman coupling coefficient) increases with the frequency, ${ }^{42}$ and consequently decreases with increasing ring size. On the same line, it has been observed that in densified silica the frequency of bending modes increases due to the decreased mean intertetrahedra $\mathrm{Si}-\mathrm{O}-\mathrm{Si}$ angles and $\mathrm{Si}-\mathrm{Si}$ distances (as a consequence of puckering), while the frequency of stretching modes is less affected by densification. ${ }^{39,42}$ To summarise, these effects, alongside line broadening due to the ring units not being perfectly planar, and the energetically close vibrations due to ring units of similar size have rendered the assignment of vibrational modes in zeolites a challenging task.

In line with previous works focused on the use of vibrational spectroscopy to characterise zeolites, we attribute the most intense feature peaked at $383 \mathrm{~cm}^{-1}$ to the most abundant ring unit that in silicalite- 1 is the 5 -membered ring. ${ }^{13}$ Accordingly, and in order of decreasing frequencies, we also assign the signature at $360 \mathrm{~cm}^{-1}$ to 6 -membered rings, whereas the one at $294 \mathrm{~cm}^{-1}$ is attributed to 10 -membered rings. The latter is in light contrast to one of the precedent works, in which the mode at $294 \mathrm{~cm}^{-1}$ was attributed to 6-membered rings instead. ${ }^{13}$ Our assignment is primarily supported by the complementary Raman spectrum recorded for the zeolite SSZ-13 (with the CHA framework structure that consists of 4-membered, 6-membered and 8-membered rings only), which is congruent with the correlation between ring size and frequency of the O-bending modes (see Fig. S1, ESI $\dagger$ ). In addition, the contribution at $360 \mathrm{~cm}^{-1}$ is evidenced here thanks to a high spectral resolution, but has otherwise been hidden under the stronger feature peaked at $383 \mathrm{~cm}^{-1}$ in precedent studies. The sharp signature at $472 \mathrm{~cm}^{-1}$ is attributed to 4-membered rings, in line with what has been proposed by other authors before. ${ }^{8,12,13,16}$ These rings are not visible on the $a-c$ plane shown in Fig. 5 since they connect different planes to each other and are thus parallel to the $a-b$ plane. It is notable that the Raman spectra shown in Fig. 6, recorded using the incident light at different polarisations but in the absence of an analyser (i.e. without a polarisation filter), show different peak intensities. That is, the feature at $472 \mathrm{~cm}^{-1}$ is emphasized when the incident light is normally polarised (i.e. polarised along the $x$-axis) while the features at 383 and $832 \mathrm{~cm}^{-1}$ are emphasised when the incident light is orthogonally polarised (i.e. polarised along the $y$-axis). The Raman spectrum recorded with the incident light having a circular polarisation shows, as expected, an intermediate intensity at these frequencies. These aspects, emphasised here for the first time, are a direct manifestation of the anisotropy of the MFI framework structure.

4.1.1 Cross polarised Raman spectra. More precise Raman measurements were performed using parallel and cross polarised conditions or, according to the Porto notation, in the $z(x x) \bar{z}$ and $z(x y) \bar{z}$ as well as in the $z(y y) \bar{z}$ and $z(y x) \bar{z}$ set ups, as

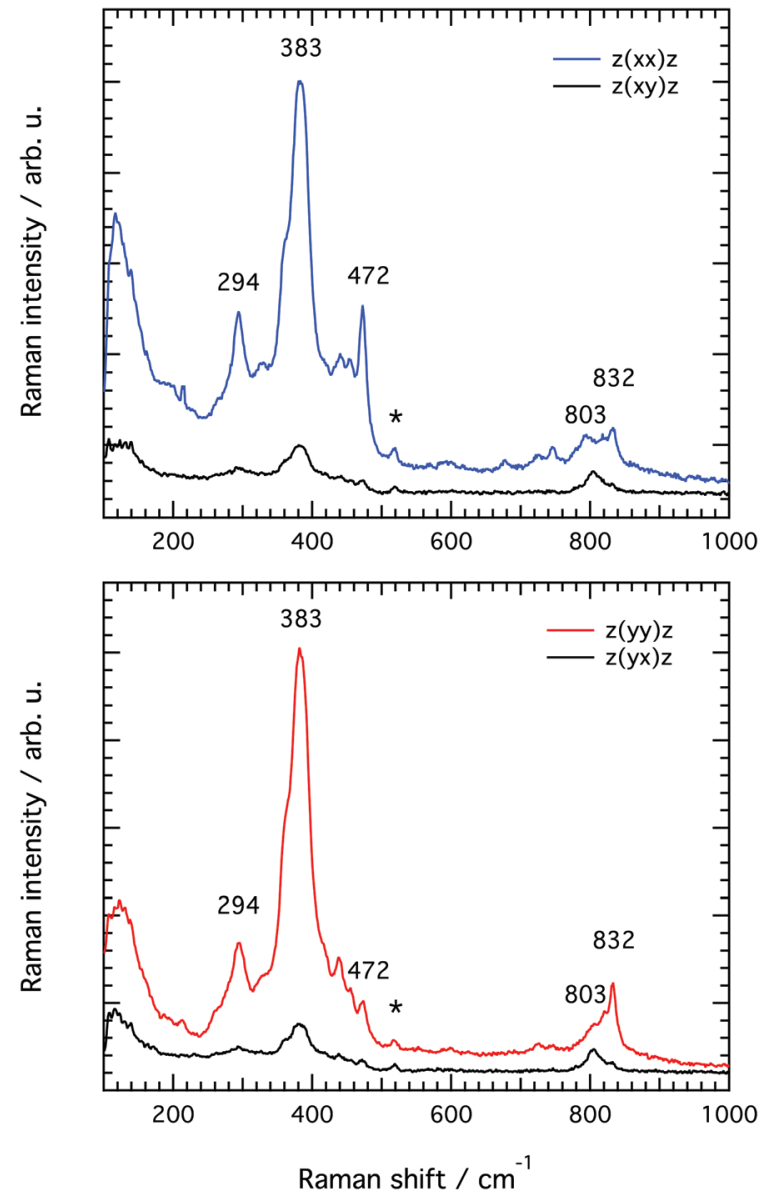

Fig. 7 Raman spectra recorded from the upper $a-c$ surface of a single crystal of silicalite- 1 at parallel (that is $z(x x) z$ and $z(y y) z$ ) and cross polarised (that is $z(x y) z$ and $z(y x) z)$ configurations. The asterisk marks a peak arising from the aluminium substrate and is thus not associated to any vibrational mode in silicalite-1.

summarised in Fig. 7. The differences observed between the spectra recorded with the $z(x x) \bar{z}$ and $z(y y) \bar{z}$ configurations confirm that structural diversity exists along the two crystal axes $a$ and $c$, and most importantly that polarised Raman spectroscopy is sensitive to these differences. Moreover, the drastic intensity decrease observed when changing the polarisation configuration from $z(x x) \bar{z}$ to $z(x y) \bar{z}$ and from $z(y y) \bar{z}$ to $z(y x) \bar{z}$ suggests that the recorded vibrations are close to totally polarised. More specifically, the depolarisation ratio $\rho$, estimated as $\rho=I_{z(x y) z} / I_{z(x x) z}$ or $\rho=I_{z(y x) z} / I_{z(y y) z}$ is found to be well below 0.75 for most vibrations, the threshold value that distinguishes polarised from depolarised vibrations, see also the calculated values given in Table 2 (each value of $\rho$ has been obtained from the ratio of integrated areas estimated from the peak fit of the Raman spectra. An example of the peak fit model used in the lower frequency range is shown in Fig. S2, ESI $\dagger$ ). An exception is the vibrational mode found by us at $803 \mathrm{~cm}^{-1}$ that shows a depolarisation ratio close to one, although this will be discussed in more detail further down. By definition, totally polarised vibrations are those that scatter light with the same polarisation as the incident light, which happens to be the case of symmetric vibrational modes. 
Table 2 Frequency of the observed vibrational modes (in $\mathrm{cm}^{-1}$ ), proposed assignment, depolarisation ratios ( $\rho$ ), type of vibration (polarised/nonpolarised), pseudo-order parameter $(f)$ and isotropy of the bond underlying the observed vibration. The symbol $\nu$ stands for stretching and the symbol $\delta$ stands for bending (here also including torsional and deformation modes), while the subscripts as and s mean anti-symmetric and symmetric respectively

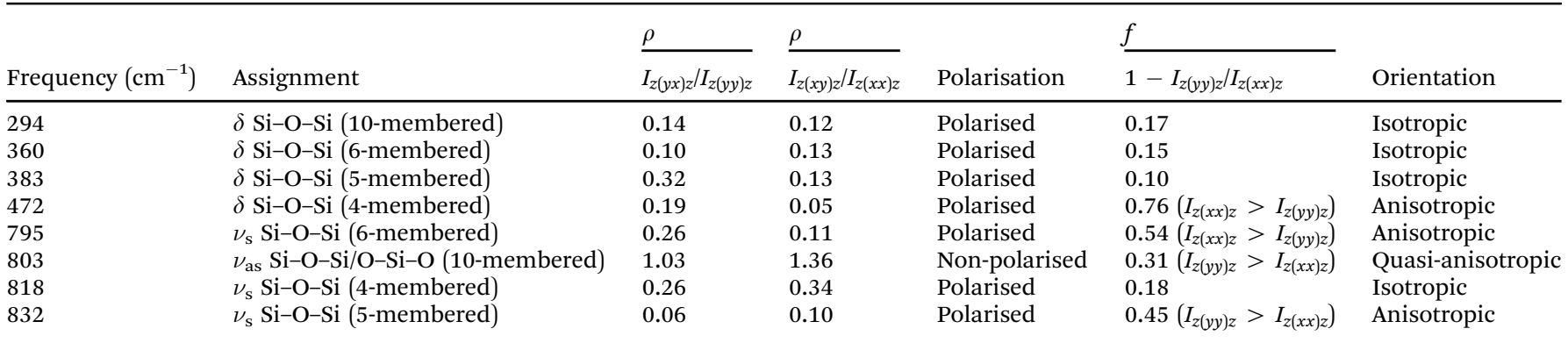

One additional information that can be extracted from using polarised Raman spectroscopy is the so called pseudo-order parameter, $f$, expressed as $f=1-\left(I_{y y} / I_{x x}\right)$ or $f=1-\left(I_{x x} / I_{y y}\right)$ depending on the orientation of the specific material and the vibration that is analysed. This parameter can be investigated for each vibrational mode independently. In isotropic materials $I_{x x}$ is expected to be equal to $I_{y y}$, which results in $f$ being equal to zero. If a specific molecular orientation exists, $f$ differs from zero and a value of one is achieved for a perfect alignment between the molecular bond underlying the vibration and the direction of polarisation of the incident light. The pseudo-order parameter has previously been estimated to achieve orientational information in several materials such as stretched synthetic polymer fibres, nanotubes and cellulose fibres. ${ }^{26-28,43}$ As also given in Table 2, we find that for the features peaked at 294, 360 and $383 \mathrm{~cm}^{-1}$ the pseudo-order parameter has a low value, between 0.10 and 0.20 , indicating that the bonds underlying these vibrations are isotropic in nature. Based on this and the small value of $\rho$, we can conclude that at these three frequencies symmetric ring opening modes contribute the most (but certainly not exclusively, since more complex modes such as deformation or torsion can also contribute and overlap. According to precedent studies on vitreous silica, however, O-bending modes seem to dominate in the spectral range below $600 \mathrm{~cm}^{-140,42}$ ). The feature at $472 \mathrm{~cm}^{-1}$ is also associated to a ring mode, but shows a clear anisotropic character $\left(f=0.76\right.$, with $\left.I_{z(x x) z}>I_{z(y y) z}\right)$. Hence, this vibrational mode includes contributions from $\mathrm{O}$ atoms whose movement is oriented along the $x$-axis (or along the $c$-axis of the crystal). Looking at the ring structure reproduced in Fig. 8, but also the more detailed structure of the pentasil pair given in Fig. 9 (reproduced with an atom labelling equivalent to that proposed by Lermer et $a l^{2}$ and Olson et $a l^{44}$ ), a plausible assignment of the $472 \mathrm{~cm}^{-1}$ mode is to $\mathrm{O}$ atoms shared between two 5-membered rings above or below a 6-membered ring (i.e. to oxygen atoms in the $\mathrm{T} 3-\mathrm{O}-\mathrm{T} 4$ bond) or to $\mathrm{O}$ atoms belonging to the 4-membered rings (i.e. to oxygen atoms in the T9-O-T10 bond). Here one should note that the spectral feature at $459 \mathrm{~cm}^{-1}$ has a similar character to that at $472 \mathrm{~cm}^{-1}$, although it has a relatively lower intensity. We propose to assign the vibration observed at $472 \mathrm{~cm}^{-1}$ to O-bending modes in 4-membered rings, with the argumentation that the T9-O-T10 bond is characterised by a smaller T-O-T angle $\left(153.2^{\circ}\right)$ than the T3-O-T4 bond $\left(176.2^{\circ}\right){ }^{2}$

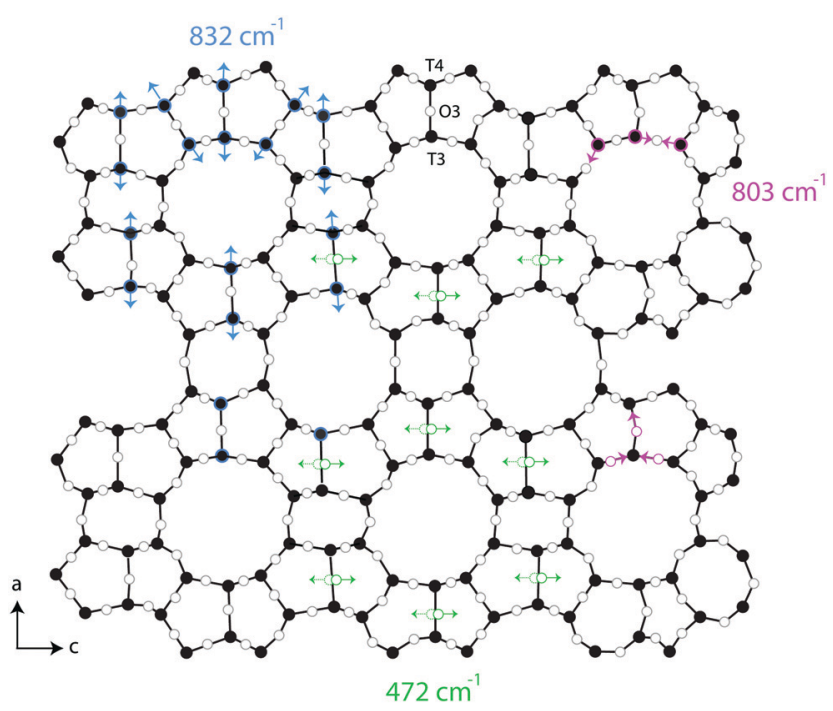

Fig. 8 Projection of the ring units seen over the $a-c$ plane of a MFI framework structure. Blue arrows indicate the $\mathrm{Si}-\mathrm{O}-\mathrm{Si}$ bonds contributing to the stretching mode observed at $832 \mathrm{~cm}^{-1}$, while green arrows mark the position of $\mathrm{O}$-atoms involved in the $\mathrm{O}$-bending modes appearing at $472 \mathrm{~cm}^{-1}$. The proposed assignment of anti-symmetric stretching modes at $803 \mathrm{~cm}^{-1}$ is also indicated.

which tends to blue shift the vibrational frequency, and that the feature is sharper $\left(\right.$ fwhm $_{472}=12 \mathrm{~cm}^{-1}$ while fwhm $\left.\mathrm{fm}_{459}=18 \mathrm{~cm}^{-1}\right)$ with sharpness being typical of planar rings; ${ }^{39,41}$ in fact the 4-membered ring is the only truly planar sub-unit in the MFI framework structure. In this case, the polarisation dependent intensity must origin from out-of-plane ring deformation modes (since the in-plane O-bending modes occur over the $a-b$ plane). We will come back to this assignment through the discussion of polar plots, but we anticipate that Raman active modes observed close to $470 \mathrm{~cm}^{-1}$ in diverse zeolitic materials have already been proposed to arise from 4-membered rings. ${ }^{8,12,13,16}$ Consistent with this assignment is the Raman spectrum that we have recorded for a zeolite with the chabazite structure (see Fig. S1, ESI $\dagger$ ), which shows two sharp and distinct peaks around $470 \mathrm{~cm}^{-1}$ in agreement with the presence of two types of 4-membered rings in the framework.

The spectral region $650-920 \mathrm{~cm}^{-1}$, where $\mathrm{Si}-\mathrm{O}-\mathrm{Si}$ and $\mathrm{O}-\mathrm{Si}-\mathrm{O}$ stretching modes are known to appear, has required a meticulous 


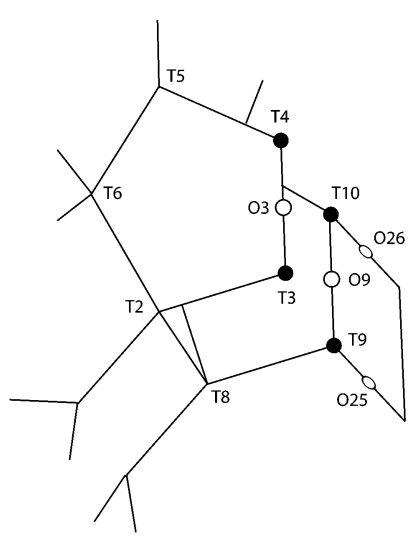

Fig. 9 The pentasil sub-unit with atom numbering as proposed by Olson et al. ${ }^{44}$ and Lermer et al. ${ }^{2}$ See also Fig. 8 for the position of this sub-unit (e.g. the location of T3 and T4) in the context of the larger structure. The T3-O3-T4 and T9-O9-T10 bonds belong to 5-membered rings placed on different planes along the $b$-axis.

analysis through careful peak fitting due to the low intensity of the modes and the proximity of several energetically close contributions. Fig. 10 illustrates the peak fit model that we have used, which between 760 and $850 \mathrm{~cm}^{-1}$ includes five Lorentzian functions. An important note is that the position and width of the fit peaks have been fixed at values obtained from the fit procedure of the spectra recorded at parallel configurations $\left(I_{x x}\right.$ and $\left.I_{y y}\right)$, while the intensity of the peaks was left as a free parameter. It is also important to mention that an alternative model with four components only (one component being free to be positioned between 795 and $803 \mathrm{~cm}^{-1}$ ) has also been tested, but resulted in inconsistent trends. Hence, despite models with fewer components are generally preferred, in this case we opted for the five peak model, which is strongly suggested by the position of the distinct local maxima observed in both the parallel and cross polarised spectra.

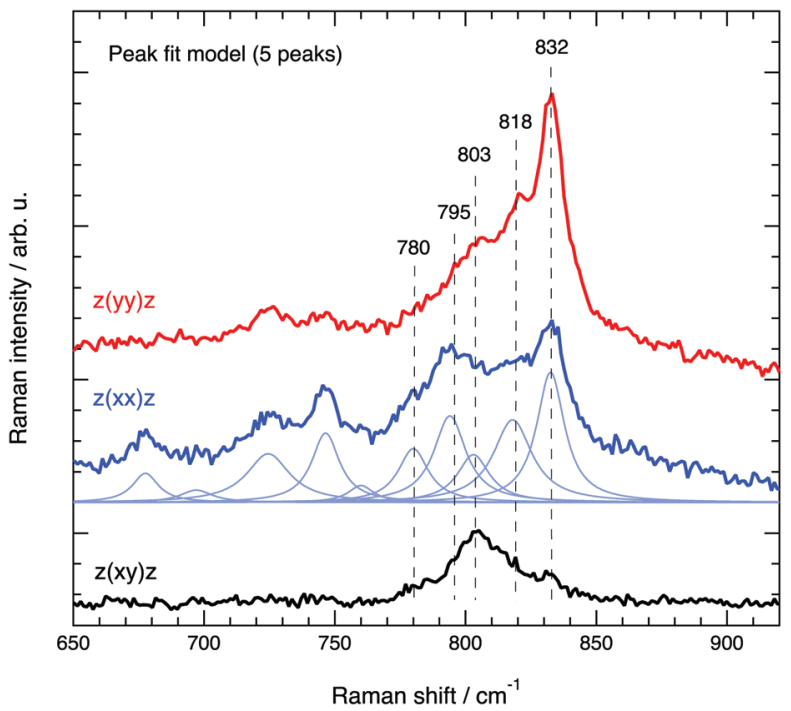

Fig. 10 Peak fit model based on a linear background and several Lorentzian functions used to fit the Raman spectra of silicalite- 1 in the range $650-920 \mathrm{~cm}^{-1}$.
For clarity, the remaining of the discussion is based on the five peaks model with components centred at 780, 795, 803, 818 and $832 \mathrm{~cm}^{-1}$.

In the past, a distinction has been made between modes observed between 650 and $730 \mathrm{~cm}^{-1}$ which were assigned to intra-tetrahedral $\mathrm{O}-\mathrm{Si}-\mathrm{O}$ stretching, and modes observed between 760 and $850 \mathrm{~cm}^{-1}$ which were assigned to inter-tetrahedral $\mathrm{Si}-\mathrm{O}-\mathrm{Si}$ stretching. ${ }^{10}$ Because internal $\mathrm{O}-\mathrm{Si}-\mathrm{O}$ angles are stiffer than the $\mathrm{Si}-\mathrm{O}-\mathrm{Si}$ bridging angles, ${ }^{38}$ the latter region (i.e. the $760-850 \mathrm{~cm}^{-1}$ range) is expected to be more sensitive to structural changes (i.e. to ring size and $\mathrm{Si}-\mathrm{O}-\mathrm{Si}$ bond angle variations), yet still less affected than the vibrations categorised as bending modes. ${ }^{42}$

A distinct behaviour is that of the component at $832 \mathrm{~cm}^{-1}$, which is also the most isolated and easily fitted feature in this region. It is clearly more intense when measuring in the $z(y y) z$ configuration than when using $z(x x) z$ (Fig. 7 and 10), has a relatively high value for the pseudo-order parameter $(f=0.45$, or $\left.I_{y y} \approx 2 \cdot I_{x x}\right)$ and a low depolarisation ratio $(\rho<0.10)$. These details indicate that the vibrational mode can be assigned to a symmetric stretching with a considerable contribution from $\mathrm{Si}-\mathrm{O}-\mathrm{Si}$ bonds oriented along the $a$-axis of the crystal. As shown in Fig. 8, these may be the bonds shared between two 5-membered rings adjacent to one 6-membered ring, marked by blue arrows in the figure, or in other words the T3-O-T4 bonds (see also Fig. 9). However, as discussed above Fig. 9 shows that in alternating layers along the $b$-axis oriented $\mathrm{Si}-\mathrm{O}-\mathrm{Si}$ bonds can also belong to the 4-membered ring unit (the T9-O9-T10 bonds). The refined crystallographic studies performed by Lermer et $a .^{2}$ and Olson et $a .^{44}$ report average $\mathrm{T}-\mathrm{O}$ bond lengths of $1.58 \AA$ for the T3-O3-T4 bond and of $1.6 \AA$ for the T9-O9-T10 bond. Since stretching frequencies are strongly related to the bond length (that is to the bond strength), we conclude that the vibrational feature at $832 \mathrm{~cm}^{-1}$ is primarily due to the stretching of $\mathrm{Si}-\mathrm{O}-\mathrm{Si}$ bonds shared between 5 -membered rings. This make sense with the associated frequency being the highest within the investigated range $760-850 \mathrm{~cm}^{-1}$.

The feature at $803 \mathrm{~cm}^{-1}$ has a different behaviour, in particular it shows a high depolarisation ratio and a relatively low pseudo-order parameter $(f=0.31)$. These details are consistent with quasi isotropic $\mathrm{Si}-\mathrm{O}-\mathrm{Si}$ and/or $\mathrm{O}-\mathrm{Si}-\mathrm{O}$ bonds and vibrations with an anti-symmetric character. This is an interesting finding since the spectral region $760-850 \mathrm{~cm}^{-1}$ is often described to only contain symmetric stretching modes, as opposed to the high frequency range around $1000 \mathrm{~cm}^{-1}$ that is typically assigned to anti-symmetric stretching. Nevertheless, in one previous study focused on amorphous silica a high depolarisation ratio for a mode close to $800 \mathrm{~cm}^{-1}$ has been reported upon comparison between cross and parallel polarised Raman spectra, ${ }^{40}$ while the contribution of anti-symmetric stretching modes in at least one of the peaks found within the range $760-850 \mathrm{~cm}^{-1}$ has also been proposed to exist. ${ }^{38}$ Our results clearly indicate that at least one vibrational mode arises from the anti-symmetric displacement of $\mathrm{O}$ or $\mathrm{Si}$ atoms. Due to a strong coupling between $\mathrm{Si}-\mathrm{O}-\mathrm{Si}$ and $\mathrm{O}-\mathrm{Si}-\mathrm{O}$ stretching modes however, and the moderate difference between the masses of $\mathrm{O}$ and $\mathrm{Si}$, the frequency of these vibrations can easily overlap and 


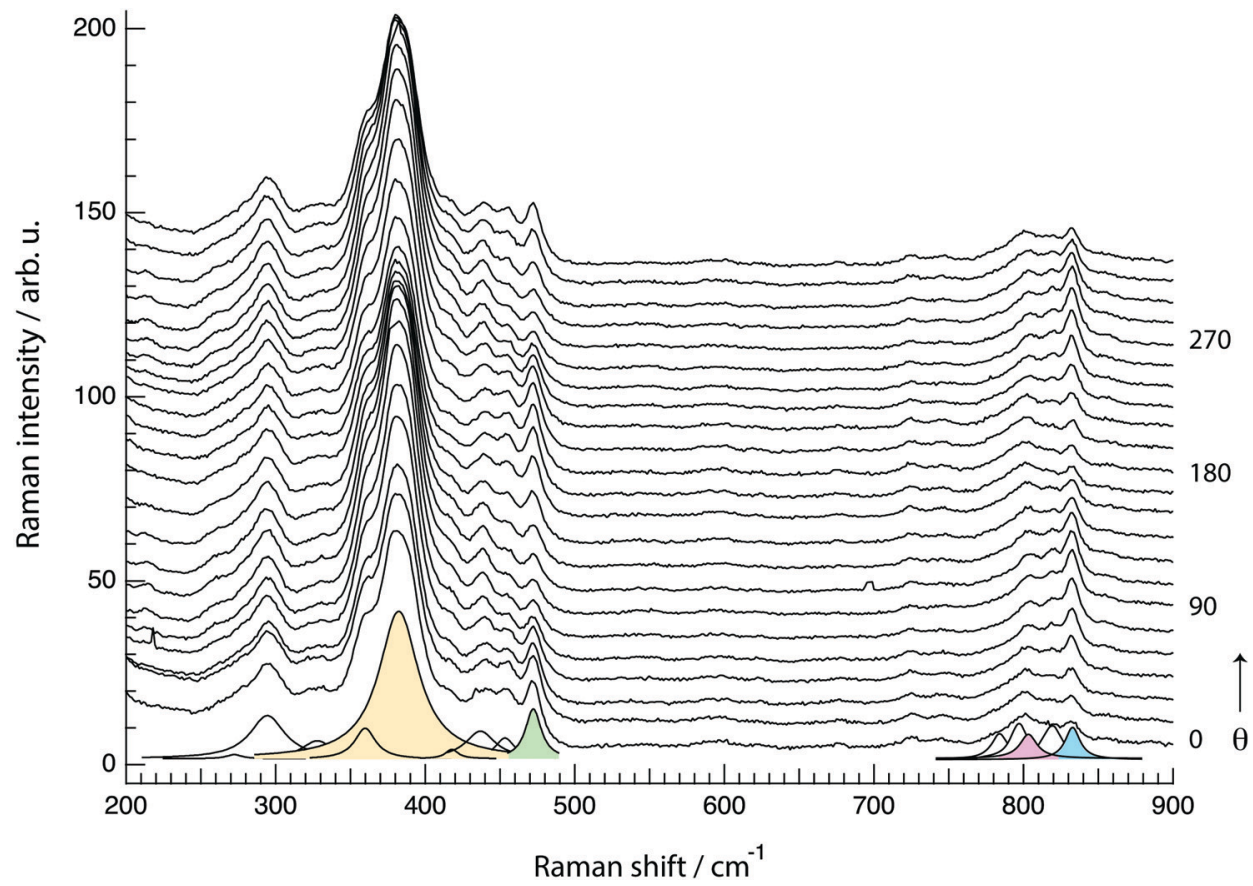

Fig. 11 A selection of Raman spectra recorded from a single crystal of silicalite-1 with the polarisation angle $\theta$ rotating around the $z$-axis and over the $x-y$ plane. The peak fit model used to analyse different spectral regions is shown at the bottom of this figure emphasising in colour particularly important components.

cannot be distinguished. A tentative assignment of the feature at $803 \mathrm{~cm}^{-1}$ is thus to anti-symmetric $\mathrm{Si}-\mathrm{O}-\mathrm{Si}$ and/or $\mathrm{O}-\mathrm{Si}-\mathrm{O}$ stretching modes in bonds belonging to 10 -membered rings. The contribution at $795 \mathrm{~cm}^{-1}$ has a low depolarisation value (below 0.26$)$ and a relatively high value of $f\left(f=0.54\right.$, with $I_{z(x x) z}>$ $\left.I_{z(y y) z}\right)$, which suggests its assignment to symmetric stretches with an orientation along the laboratory $x$-axis (or along the $c$-axis of the crystal). Plausible bonds that can contribute at this frequency are $\mathrm{Si}-\mathrm{O}-\mathrm{Si}$ and/or $\mathrm{O}-\mathrm{Si}-\mathrm{O}$ stretching modes in 6-membered rings, given that precedent crystallographic studies have already evidenced a flatter contour for this ring unit. ${ }^{1}$

4.1.2 Angle dependent Raman spectra. In order to better understand the character of the observed Raman active vibrational modes, angle dependent Raman measurements have been performed keeping the sample fixed and rotating the polarisation of the incident light from $0^{\circ}$ to $360^{\circ}$ at steps of $2^{\circ}$. With the set up used (see Fig. 3), $0^{\circ}$ is equivalent to having the polarisation parallel to the $x$ - or $c$-axis, while $90^{\circ}$ is equivalent to having the polarisation parallel to the $y$-or the $a$-axis. No analyser was used in this set of measurements and light scattered with a polarisation at all directions could be collected. Fig. 11 shows a selection of the collected spectra, vertically offset for a clearer illustration of the angular dependence. Raman intensities have then been extracted from every single spectrum as integrated areas obtained from the peak fit analysis, as shown by the coloured components in the bottom most part of the figure. The Raman intensities so obtained are shown in linear plots in the top panel of Fig. 12 as a function of the rotation angle $\theta$, and reveal an angular dependence for all the vibrational modes with a periodicity close to $90^{\circ}$, which is consistent with the orthogonality of the crystal's axes. However, while the features at 383 and $832 \mathrm{~cm}^{-1}$ show maxima at $90^{\circ}$ and $270^{\circ}$, the one at $472 \mathrm{~cm}^{-1}$ has its maxima at $0^{\circ}$ and $180^{\circ}$, and the feature at $803 \mathrm{~cm}^{-1}$ shows an almost angle-independent behaviour. These trends are better visualised in the polar plots, see mid panel of Fig. 12. To rationalise these differences one must compare the experimental results with simulated plots, which require the use of real values for the Raman tensors. The tensor coefficients could be achieved from the cross-polarised measurements ${ }^{45}$ and resulted to be, after internal normalisation, as follows:

$$
\begin{aligned}
& 383 \mathrm{~cm}^{-1}:\left(\begin{array}{ll}
0.95 & 0.32 \\
0.31 & 1.00
\end{array}\right) \quad 472 \mathrm{~cm}^{-1}:\left(\begin{array}{ll}
1.00 & 0.22 \\
0.23 & 0.50
\end{array}\right) \\
& 803 \mathrm{~cm}^{-1}:\left(\begin{array}{ll}
0.83 & 0.97 \\
1.00 & 1.00
\end{array}\right) \quad 832 \mathrm{~cm}^{-1}:\left(\begin{array}{ll}
0.74 & 0.26 \\
0.28 & 1.00
\end{array}\right)
\end{aligned}
$$

The fact that $\alpha_{x x} \neq \alpha_{y y}$ in the case of e.g. the features at 472 and $832 \mathrm{~cm}^{-1}$ reflects, once again, the anisotropic character of the underlying vibrational modes (a more complete set of tensor coefficients including also other vibrational modes can be found in the ESI $\dagger$ file, page 2).

The angular dependence of the feature at $383 \mathrm{~cm}^{-1}$ has been simulated considering the vibrational mode as symmetric (due to the character of its Raman tensor) and hence expected to have an intensity proportional to $\left(0.95 \cos ^{2} \theta+1 \sin ^{2} \theta\right)^{2}+$ $(1-0.95)^{2} \cos ^{2} \theta \sin ^{2} \theta$, i.e. as the sum of eqn (1) and (3) since no analyser was used (see the Theoretical aspects section in the beginning of this article). This simulated angular dependence 

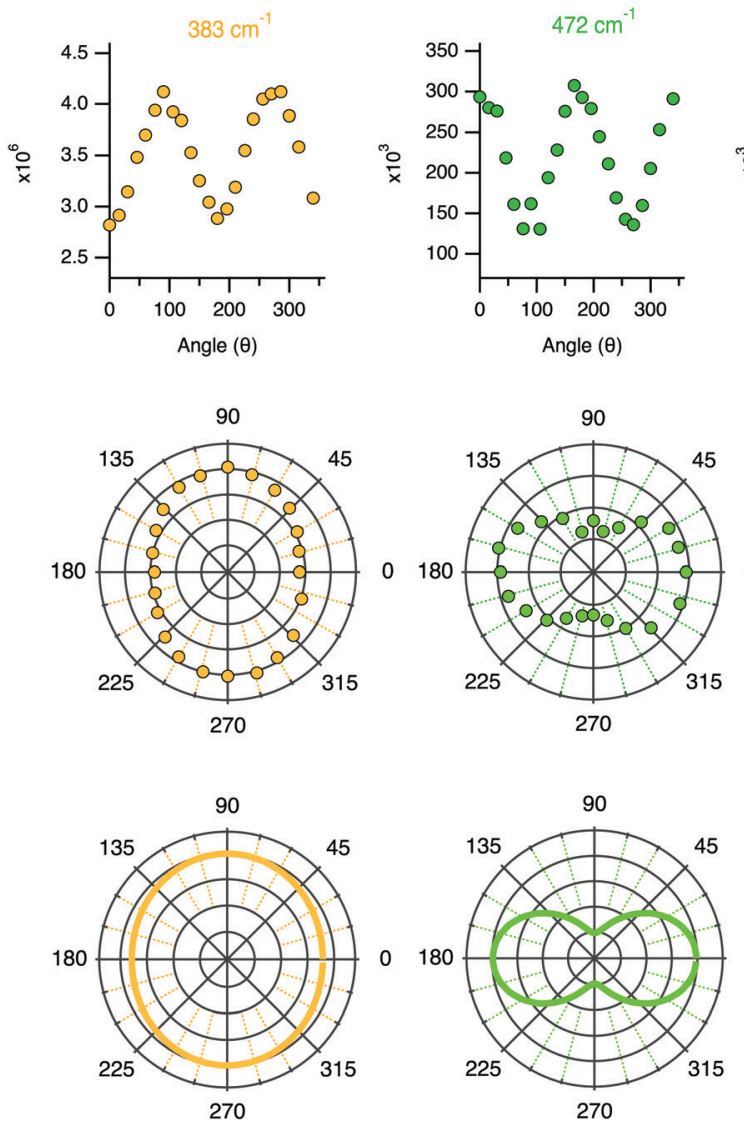
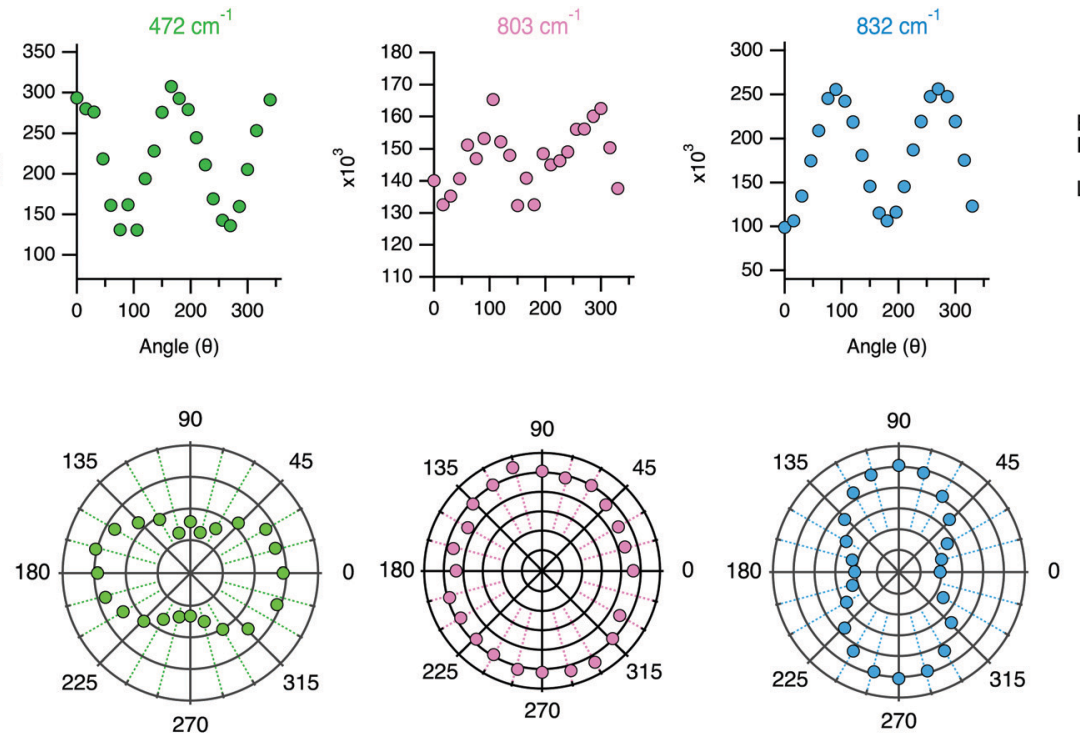

Experimental

Raman intensities

Polar plots

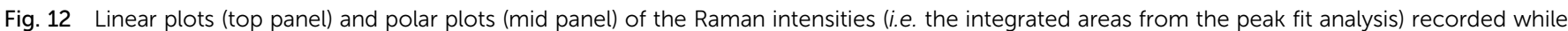

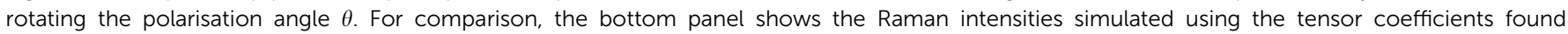
experimentally and using either eqn (1) and (3) or eqn (2) and (4) depending on the character of the vibration.

is shown in the bottom row of Fig. 12 and reveals a fairly good agreement with the behaviour of the experimental data. Nevertheless, a better match is obtained if the coefficient $\alpha_{x x}$ in the equation above is reduced to 0.90 (see Fig. S3, ESI $\dagger$ ). The simulated angular dependence for the vibrational mode at $472 \mathrm{~cm}^{-1}$ has also been reproduced assuming a symmetric character, but now the expression $\left(1 \cos ^{2} \theta+0.5 \sin ^{2} \theta\right)^{2}+$ $(1-0.5)^{2} \cos ^{2} \theta \sin ^{2} \theta$ results in a double loop shape with minima at 90 and $270^{\circ}$. The agreement between the simulation and the experiments is, in this case, very good. This confirms the symmetric character of the vibrational mode, which we thus assign to the in-phase out-of-plane deformation mode in 4-membered rings. An almost perfect match is also found between the experimental polar plots for the feature at $832 \mathrm{~cm}^{-1}$ and the polar plots simulated using the coefficients $\alpha_{x x}=0.74$ and $\alpha_{y y}=1.00$, and thus expecting the Raman intensity to be proportional to $\left(0.74 \cos ^{2} \theta+1 \sin ^{2} \theta\right)^{2}+(1-0.74)^{2} \cos ^{2} \theta \sin ^{2} \theta$. The maxima at $90^{\circ}$ and $270^{\circ}$ and the overall shape of the loop are both very well reproduced, confirming that the vibrational mode contributing at this frequency is an anisotropic symmetric stretching. The experimental angular dependence of the integrated area under the feature peaked at $803 \mathrm{~cm}^{-1}$ is weak, with marginally higher intensities at $0^{\circ}$ and $180^{\circ}$. Neither by treating the vibration as purely symmetric (i.e. as the sum of eqn (1) and (3)) nor by treating it as anti-symmetric (i.e. by the sum of eqn (2) and (4)) could the angular dependent experimental data be well reproduced using the found values of the Raman tensor. By contrast, a weighted sum with $80 \%$ of anti-symmetric character results in a good fit to the data, which is also the simulated behaviour shown in Fig. 12. This approach is motivated by the high values of the off-diagonal coefficients, which is typical for anti-symmetric vibrations. This finding is in agreement with the conclusions drawn from cross polarised measurements (Section 4.2.1), and supports the predicted presence of anti-symmetric stretching modes within the range $760-850 \mathrm{~cm}^{-1} \cdot 38,40$

An interesting observation is that while the polar plot of the $294 \mathrm{~cm}^{-1}$ is rather symmetric with respect to $\theta$, the polar plots for the 360 and $383 \mathrm{~cm}^{-1}$ modes show a similar shape with slightly higher intensities at $90^{\circ}$ and $270^{\circ}$ (see Fig. S4, ESI $\dagger$ ). This could be explained by the flatter contour of the 6-membered ring structures along the $c$-axis, as also illustrated in Fig. 8. Also, the fact that the polar plots of the peaks at $472 \mathrm{~cm}^{-1}$ and $832 \mathrm{~cm}^{-1}$ are orthogonal to each other, is in complete agreement with the assignment of the vibrational mode at $472 \mathrm{~cm}^{-1}$ to the motion of $\mathrm{O}$ atoms belonging to 4-membered rings. As can be seen from the 3D structural reconstruction of the MFI framework from crystallographic data (Fig. 9 and/or www.europe.ize.org) the 4-membered rings are in fact oriented orthogonally to the 5-membered rings 


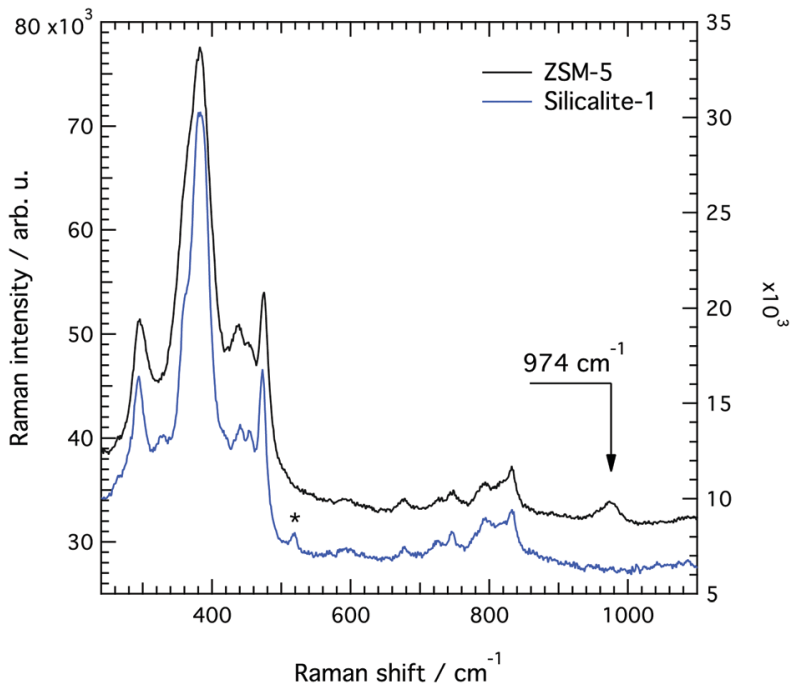

Fig. 13 Raman spectra collected from single crystals of silicalite-1 (blue) and ZSM-5 (dark grey) with the polarisation of the incident light parallel to the $c$-axis in both cases. A new feature is observed at $974 \mathrm{~cm}^{-1}$ in ZSM-5. The asterisk indicates a signature arising from the aluminium foil underlying the single crystal.

appearing on the $a-c$ plane, which implies that the motion of the $\mathrm{O}$ atoms during the 4-membered ring deformation mode is indeed perpendicular to the displacement of $\mathrm{Si}$ atoms during $\mathrm{Si}-\mathrm{O}-\mathrm{Si}$ stretching modes contributing at $832 \mathrm{~cm}^{-1}$.

4.1.3 Single crystals of ZSM-5. The Raman spectrum recorded from a single crystal of ZSM-5 with a SAR equal to 50 is equivalent, but not identical, to that recorded for silicalite-1, Fig. 13. This is true with respect to absolute frequencies and relative intensities, which reflects a perfect integrity of the framework structure of the zeotype. The overall angular dependence of Raman intensities in silicalite-1 and ZSM-5 are shown in Fig. S5 and S6 (ESI $\dagger$ ) respectively, as linear and polar plots. These intensities were obtained by the software (Wire 5.0) as 'intensity-to-baseline' values, which is a less accurate approach than by peak fitting every single spectrum but equally informative. The plots show a similar angular behaviour (with slightly less marked minima in the case of ZSM-5) and confirm that the symmetry and anisotropic character of the zeotype is independent on the inclusion of $\mathrm{Al}$ atoms in the framework structure, at least for the SAR considered in this study.

A difference between the Raman spectra of silicalite- 1 and ZSM-5 is the width of the peaks, ZSM-5 showing overall broader spectral features. The feature at about $360 \mathrm{~cm}^{-1}$, for example, appears less defined because of its broader character. Line broadening is consistent with an increased disorder in the local structure due to the presence of $\mathrm{Al}$ atoms at a low ratio. As a note, structures with $100 \%$ of $\mathrm{Si}$ atoms, $100 \%$ of $\mathrm{Al}$ atoms or $50 \%$ of $\mathrm{Si}$ and $\mathrm{Al}$ atoms would all re-establish order. From a careful peak fit analysis (see Fig. S7, ESI $\dagger$ ) we find that the feature at $472 \mathrm{~cm}^{-1}$ is less affected (i.e. is broadened the least) which indicates that the 4-membered ring is a less probable site for the $\mathrm{Al}$ atoms.

The most important difference to the spectrum of silicalite-1 is, however, a new vibrational mode at $974 \mathrm{~cm}^{-1}$, whose assignment is a subject of debate. Given the spectral region where it appears, this mode could a priori arise from in-plane AlO-H bending, Al-O(H)-Si stretching or Al-OH stretching. A vibration at or close to this frequency has also been observed in some other $\mathrm{Al}$ containing zeolites. ${ }^{13,16,18}$ In particular, Mofrad et al. ${ }^{18}$ have reported the trend of Raman frequencies theoretically calculated for sodalite (that adopts the SOD framework structure) at decreasing SAR values, highlighting an increasing contribution close to $970 \mathrm{~cm}^{-1}$. This was interpreted to arise from a red shift of the $\mathrm{T}-\mathrm{O}-\mathrm{T}$ anti-symmetric stretching modes otherwise found between 1000 and $1200 \mathrm{~cm}^{-1}$, an assignment that is supported by the fact that $\mathrm{Al}-\mathrm{O}$ bonds are expected to be weaker than $\mathrm{Si}-\mathrm{O}$ bonds. For example, according to the experimental work of Yu-Ran Luo, ${ }^{46}$ the bond dissociation energy is lower in $\mathrm{Al}-\mathrm{O}\left(501.9 \mathrm{~kJ} \mathrm{~mol}^{-1}\right)$ than in $\mathrm{Si}-\mathrm{O}\left(799.6 \mathrm{~kJ} \mathrm{~mol}^{-1}\right)$, if diatomic molecules are considered.

The tensor coefficients found by us from cross polarised measurements for the $974 \mathrm{~cm}^{-1}$ mode are $\alpha_{x x}=0.88, \alpha_{y y}=1$, $\alpha_{x y}=0.32$ and $\alpha_{y x}=0.30$, which is in contrast with the supposed anti-symmetric character of the vibration. Moreover, the dependence on the rotation angle $\theta$ of the integrated area under this mode (see Fig. 14, left panel) reveals a weak anisotropy, similarly to the case of the vibrational mode at $832 \mathrm{~cm}^{-1}$ but less pronounced. The theoretical angular behaviour calculated with the tensor values $\alpha_{x x}=0.88$ and $\alpha_{y y}=1$ and assuming a symmetric character for the vibration is superimposed to the experimentally found behaviour, revealing a fit of acceptable quality. These results altogether suggest that the signature at $974 \mathrm{~cm}^{-1}$ is most likely due to $\mathrm{Al}-\mathrm{OH}$ stretching with the $\mathrm{Al}$ atom sitting at positions shared between 5-membered rings. Sites shared with six-membered rings are less probable since these would have shown an anisotropy with enhanced intensities at $0^{\circ}$ and $180^{\circ}$ (i.e. along the $c$-axis of the crystal). The assignment to Al-OH stretching is in analogy to the well known case of amorphous silica, for which a spectral feature at $980 \mathrm{~cm}^{-1}$ is typically assigned to $\mathrm{Si}-\mathrm{O}$ stretching in terminal or defect $\mathrm{Si}-\mathrm{OH}$ groups.

We have also recorded complementary Raman spectra using the $514 \mathrm{~nm}$ laser line that when coupled with the appropriate grating (2400 lines per $\mathrm{mm}$ ) allows catching the $\mathrm{O}-\mathrm{H}$ stretching

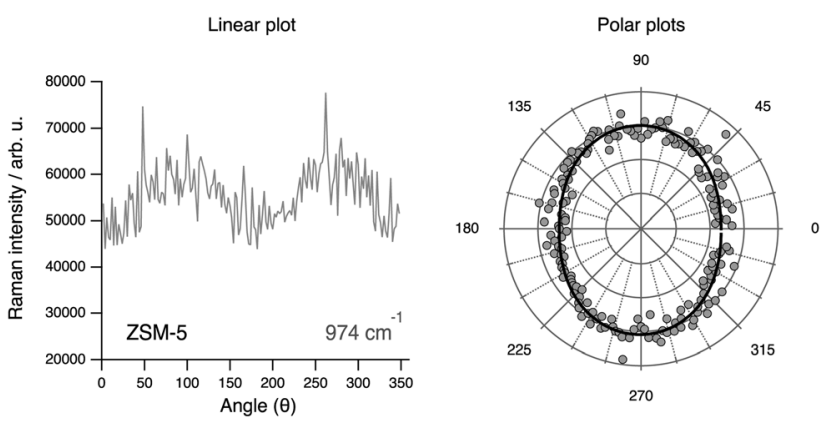

Fig. 14 Angular dependence of the area under the peak at $974 \mathrm{~cm}^{-1}$ in a linear plot (left) and in polar plots (right). In the right plot experimental values are shown as filled grey dots, while the simulated plot is shown as a solid black line. 


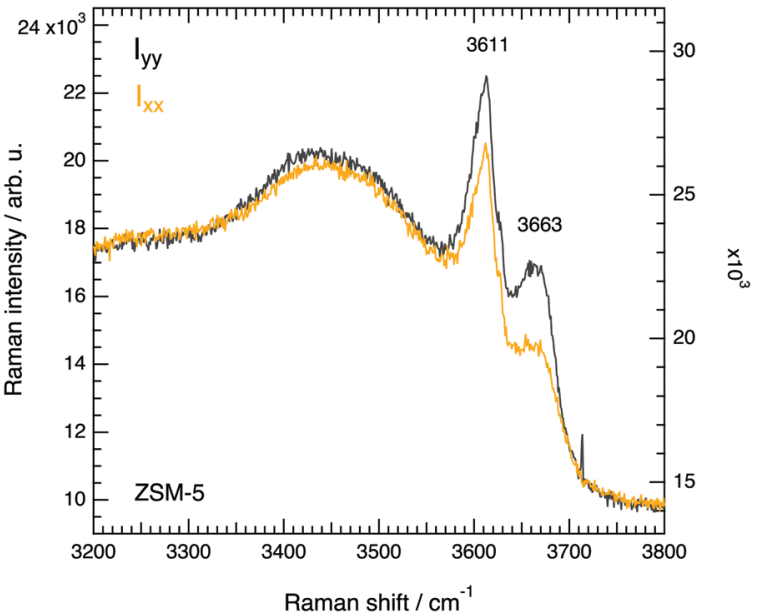

Fig. 15 High frequency range of Raman spectra recorded with the $514 \mathrm{~nm}$ laser line at parallel conditions with the polarisation of the incident laser parallel to the $c$-axis $\left(I_{x x}\right)$ or the a-axis $\left(I_{y y}\right)$ of the crystal.

modes appearing in the $3100-3800 \mathrm{~cm}^{-1}$ spectral range, see Fig. 15. Two sharp peaks are resolved, one at $3611 \mathrm{~cm}^{-1}$ which is well known to arise from the Brønsted acid sites that are of utmost importance for the catalytic reactions in ZSM- $5,{ }^{47}$ and another one at $3663 \mathrm{~cm}^{-1}$ which in infrared spectra appears as an extremely weak and broad feature and has as such rarely been reported or discussed. Nevertheless, in the recent work of Bräuer et $a .^{48}$ a feature observed at $3660 \mathrm{~cm}^{-1}$ by diffuse reflectance infrared Fourier transformed spectroscopy has been assigned to $\mathrm{O}-\mathrm{H}$ stretching modes associated to $\mathrm{Al}$ species at extra-framework positions in the internal parts of the ZSM-5. This is a plausible assignment that, if correct, would suggest that the modes at $974 \mathrm{~cm}^{-1}$ and at $3663 \mathrm{~cm}^{-1}$ arise from the same molecular group. Future spectroscopic studies, desirably at high confocal mode and through the space of single crystals, could be performed with the aim to spatially resolve, and possibly distinguish, internal from external hydroxyl groups. The features at $3611 \mathrm{~cm}^{-1}$ and $3663 \mathrm{~cm}^{-1}$ both show a lower intensity when the polarisation of the laser is set parallel to the $c$-axis of the crystal, and thus reveal a most probable orientation of the $\mathrm{O}-\mathrm{H}$ bond along the $a$-axis. Also, the intensity of the higher frequency feature appears to be more sensitive to the direction of polarisation of the incident light, $I_{x x} / I_{y y}$ being 0.8 for the peak at $3611 \mathrm{~cm}^{-1}$ and 0.6 for the peak at $3663 \mathrm{~cm}^{-1}$. This finding is pivotal and is in agreement with a recent work based on solid state NMR spectroscopy that has demonstrated a nonrandom distribution of $\mathrm{Al}$ sites within the ZSM-5 framework structure. ${ }^{4}$ The authors identify four most probable sites for the $\mathrm{Al}$ atoms, i.e. $\mathrm{T} 1$ and $\mathrm{T} 13$, and $\mathrm{T} 4$ and $\mathrm{T} 16$, which exhibit a pseudomirror symmetry. These pair of sites are spectroscopically equivalent. The striking insight is that if the $\mathrm{Al}$ atom is at position T4 (see also Fig. $2 \mathrm{~b}$ in ref. 4 ) the acidic OH group will have a preferred orientation along the $a$-axis, in agreement with the results presented in Fig. 15. We propose that further experimental work and DFT calculations will elucidate on the specific sites for the $\mathrm{Al}$ atom, possibly clarifying whether the two signatures observed in the high-frequency Raman spectrum correspond to the two most probable crystallographic sites identified by solid state NMR.

\section{Conclusions}

In this work we demonstrate that the intrinsic anisotropy in zeolites of the MFI framework structure can be studied by polarised Raman spectroscopy, provided the study is done on isolated single crystals. In other words, some Raman active modes display enhanced intensity due to a preferential orientation in space of the underlying molecular bond, as in the case of the stretching mode at $832 \mathrm{~cm}^{-1}$ and the bending mode at $472 \mathrm{~cm}^{-1}$. By using information from X-ray diffraction on the crystal's axes and the orientation of the ring units, as well as argumentations related to T-O-T bond length and angles, these modes have been assigned to have contributions from T-O-T bonds shared between two 5-membered rings $\left(832 \mathrm{~cm}^{-1}\right)$ and belonging to 4 -membered rings $\left(472 \mathrm{~cm}^{-1}\right)$. Similarly, the other Raman active modes could be assigned more precisely than proposed before thanks to detailed information achieved on isotropy and depolarisation, with strong support from the angular dependence of Raman intensities. The equivalent spectra of silicalite-1 and ZSM-5 and their almost identical behaviour upon change of polarisation of the incident light indicate that the observed vibrational modes can be assigned on the basis of local structure and bond orientation, and that none of the modes in the region $200-1400 \mathrm{~cm}^{-1}$ can be associated solely to the presence of $\mathrm{Al}$ atoms. That is, the expected blue shift of $e$.g. a stretching mode due to the lighter mass of $\mathrm{Al}$ (as compared to $\mathrm{Si}$ ) is compensated by the red shift that arises from the longer Al-O bond, which explains why the Raman spectra of ZSM-5 (with SAR $=50$ ) and silicalite- 1 are in practice identical. The only mode that distinguishes the two zeotypes is found at $974 \mathrm{~cm}^{-1}$, which we attribute to Al-OH species. This, as opposed to all the other modes, can be used in future studies as an indicator for the presence of the $\mathrm{Al}$ atom. This work also shows that the strong Raman signature at $472 \mathrm{~cm}^{-1}$ can be used as an indicator of the crystal orientation, which can be a crucial tool in the in-depth investigation of single crystals and their sub-units. Raman spectra recorded with the incident light at different polarisations also suggest that the Brønsted acid sites have a preferred orientation with respect to the crystal's axes, which seems to be in agreement with the nonrandom distribution of Al sites in ZSM-5 revealed recently by solid state NMR spectroscopy. We foresee these results to have a large impact in the field of catalysis, since the use of polarised Raman spectroscopy is a priori applicable to any zeolitic framework structure provided that single crystals several micrometers in size can be synthesised and isolated. Complementary structural and orientational knowledge from X-ray diffraction data definitely help in the assignment of the vibrational modes.

\section{Conflicts of interest}

The authors have no conflicts to declare. 


\section{Acknowledgements}

A. M. and S. V. acknowledge funding from the Knut \& Alice Wallenberg Foundation (an academy fellow grant, grant no. 2016-0220) and the Swedish Foundation for Strategic Research (SSF FFL-6, grant no. FFL-15 0092). S. C., P.-A. C. and M. S. acknowledge funding from the Knut \& Alice Wallenberg Foundation (project grant, grant no. 2015-0058) as well as from the Swedish Research Council (VR, grant no. 2017-04811).

\section{References}

1 I. Diaz, E. Kokkoli, O. Terasaki and M. Tsapatsis, Surface Structure of Zeolite (MFI) Crystals, Chem. Mater., 2004, 16(25), 5226-5232.

2 H. Lermer, M. Draeger, J. Steffen and K.-K. Unger, Synthesis and structure refinement of ZSM-5 single crystals, Zeolites, 1985, 5(3), 131-134.

3 J. Lu, E. Bartholomeeusen, B. F. Sels and D. Schryvers, Internal architecture of coffin-shaped ZSM-5 zeolite crystals with hourglass contrast unravelled by focused ion beamassisted transmission electron microscopy, J. Microsc., 2017, 265(1), 27-33.

4 E. Dib, T. Mineva, E. Veron, V. Sarou-Kanian, F. Fayon and B. Alonso, ZSM-5 Zeolite: Complete Al bond connectivity and implications on structure formation from solid-state NMR and quantum chemistry calculations, J. Phys. Chem. Lett., 2018, 9(1), 19-24.

5 A. Zheng, L. Chen, J. Yang, M. Zhang, Y. Su, Y. Yue, C. Ye and F. Deng, Combined DFT Theoretical Calculation and Solid-State NMR Studies of Al Substitution and Acid Sites in Zeolite MCM-22, J. Phys. Chem. B, 2005, 109(51), 24273-24279.

6 Z. Zhao, Y. Xing, S. Li, X. Meng, F.-S. Xiao, R. McGuire, A.-N. Parvulescu, U. Müller and W. Zhang, Mapping Al Distributions in SSZ-13 Zeolites from ${ }^{23} \mathrm{Na}$ Solid-State NMR Spectroscopy and DFT Calculations, J. Phys. Chem. C, 2018, 122(18), 9973-9979.

7 V. A. Maroni, Analysis of the vibrational characteristics of zeolites using factor group methods, Appl. Spectrosc., 1988, 42(3), 487-493.

8 P. K. Dutta, D. C. Shieh and M. Puri, Correlation of framework Raman bands of zeolites with structure, Zeolites, 1988, 8(4), 306-309.

9 P. K. Dutta, K. M. Rao and J. Y. Park, Correlation of Raman spectra of zeolites with framework architecture, J. Phys. Chem., 1991, 95(17), 6654-6656.

10 A. J. M. de Man and R. A. van Santen, The relation between zeolite framework structure and vibrational spectra, Zeolites, 1992, 12(3), 269-279.

11 B. Mihailova, V. Engström, J. Hedlund, A. Holmgren and J. Sterte, Vibrational spectroscopy study of the structure of silicalite-1 films on a gold surface, Microporous Mesoporous Mater., 1999, 32(3), 297-304.

12 E. L. Uzunova and G. St. Nikolov, Vibrational Modes of Double Four-Member Rings of Oxygen-Bridged Silicon and Aluminum Atoms: A DFT Study, J. Phys. Chem. B, 2000, 104(31), 7299-7305.
13 Y. Yu, G. Xiong, C. Li and F.-S. Xiao, Characterization of aluminosilicate zeolites by UV Raman spectroscopy, Microporous Mesoporous Mater., 2001, 46(1), 23-34.

14 H. Mikosch, E. L. Uzunova and G. St. Nikolov, Vibrational Modes of Double Six-Membered Rings of Oxygen-Bridged Silicon and Aluminum Atoms in Zeolites: A DFT Study, J. Phys. Chem. B, 2004, 108(35), 13200-13207.

15 A. B. Mukhopadhyay, C. Oligschleger and M. Dolg, Lowfrequency vibrational excitations in zeolite ZSM-5 and its partially crystalline derivatives, Phys. Rev. B: Condens. Matter Mater. Phys., 2004, 69(1), 012202.

16 B. Mihailova, V. Valtchev, S. Mintova, A.-C. Faust, N. Petkov and T. Bein, Interlayer stacking disorder in zeolite beta family: a Raman spectroscopic study, Phys. Chem. Chem. Phys., 2005, $7(14), 2756-2763$.

17 S. Bordiga, C. Lamberti, F. Bonino, A. Travert and F. ThibaultStarzyk, Probing zeolites by vibrational spectroscopy, Chem. Soc. Rev., 2015, 44(20), 7262-7341.

18 A. M. Mofrad, C. Peixoto, J. Blumeyer, J. Liu, H. K. Hunt and K. D. Hammond, Vibrational Spectroscopy of Sodalite: Theory and Experiments, J. Phys. Chem. C, 2018, 122(43), 24765-24779.

19 More explicitly, in that paper he wrote ". . .correct assignment of Raman active modes (even if the expected total number was actually observed) would be a near-to-impossible task, unless information of the type obtained from oriented single crystal laser Raman measurements were available".

20 C. Brémard and J. Laureyns, Polarized Single-Crystal MicroRaman Spectroscopy of As-Synthesized Silicalite-1, Al MFI- and Fe MFI-Type Zeolites, J. Raman Spectrosc., 1996, 27(6), 439-445.

21 C. Brémard and D. Bougeard, Raman scattering in zeolites and molecular sieves, Adv. Mater., 1995, 7(1), 10-25.

22 I. Vergilov and V. Valtchev, Raman spectra of ZSM-39 single crystals, Zeolites, 1991, 11(4), 387-390.

23 V. V. Poborchii, A. V. Kolobov, J. Caro, V. V. Zhuravlev and K. Tanaka, Polarized Raman spectra of selenium species confined in nanochannels of $\mathrm{AlPO}_{4}-5$ single crystals, Chem. Phys. Lett., 1997, 280(1-2), 17-23.

24 W. Guo, D. Wang, J. Hu, Z. K. Tang and S. Du, Raman spectroscopy of iodine molecules trapped in zeolite crystals, Appl. Phys. Lett., 2011, 98, 043105.

25 M. Tsuboi, J. M. Benevides and G. J. Thomas Jr., Raman tensors and their application in structural studies of biological systems, Proc. Jpn. Acad., Ser. B, 2009, 85(3), 83-97.

26 S. Frisk, R. M. Ikeda, D. B. Chase and J. F. Rabolt, A Determination of the Molecular Orientation of Poly(propylene terephthalate) Fibers Using Polarized Raman Spectroscopy: A Comparison of Methods, Appl. Spectrosc., 2004, 58(3), 279-286.

27 M. Tanaka and R. J. Young, Polarised Raman Spectroscopy for the study of molecular orientation distributions in polymers, J. Mater. Sci., 2006, 41(3), 963-991.

28 V. Sereda, N. M. Ralbovsky, M. C. Vasudev, R. R. Nalk and I. K. Lednev, A Polarized Raman Spectroscopy for Determining the Orientation of di-D-phenylalanine Molecules in a Nanotube, J. Raman Spectrosc., 2016, 47(9), 1056-1062.

29 X. Xu, Q. Song, H. Wang, P. Li, K. Zhang, Y. Wang, K. Yuan, Z. Yang, Y. Ye and L. Dai, In-Plane Anisotropies of Polarized 
Raman Response and Electrical Conductivity in Layered Tin Selenide, ACS Appl. Mater. Interfaces, 2017, 9(14), 12601-12607.

30 U. Ramabadran and B. Roughani, Intensity analysis of polarized Raman spectra for off axis single crystal silicon, Mater. Sci. Eng., B, 2018, 230, 31-42.

31 A. Congeduti, M. Nardone and P. Postorino, Polarized Raman spectra of a single crystal of iodine, Chem. Phys., 2000, 256(1), 117-123.

32 R. Loudon, The Raman effect in crystals, Adv. Phys., 2001, 50(7 Spec.), 813-864.

33 Ö. Attila, H. E. King, F. Meirer and B. M. Weckhuysen., 3D Raman Spectroscopy of Large Zeolite ZSM-5 Crystals, Chem. Eur. J., 2019, 25, 7158-7167.

34 N. R. Camara, V. T. Phuoc, I. Monot-Laffez and M. Zaghrioui, Polarized Raman scattering on single crystals of rare earth orthochromite $\mathrm{RCr}=3(\mathrm{R}=\mathrm{La}, \mathrm{Pr}, \mathrm{Nd}$, and $\mathrm{Sm}), J$. Raman Spectrosc., 2017, 48(12), 1839-1851.

35 R. Szostak, V. Nair and T. Thomas, Incorporation and stability of iron in molecular-sieve structures. Ferrisilicate analogues of zeolite ZSM-5, J. Chem. Soc., Faraday Trans. 1, 1987, 83(2), 487-494.

36 E. M. Flanigen, J. M. Bennett, R. W. Grose, J. P. Cohen, R. L. Patton, R. M. Kirchner and J. V. Smith, Silicalite, a new hydrophobic crystalline silica molecular sieve, Nature, 1978, 271(5645), 512-516.

37 www.cryst.ehu.es.

38 G. E. Walrafen, Y. C. Chu and M. S. Hokmabadi, Raman spectroscopic investigation of irreversibly compacted vitreus silica, J. Phys. Chem., 1990, 92(12), 6987-7002.

39 J. Burgin, C. Guillon, P. Langot, F. Vallee, B. Hehlen and M. Foret, Vibrational modes and local order in permanently densified silica glasses: Femtosecond and Raman spectroscopy study, Phys. Rev. B: Condens. Matter Mater. Phys., 2008, 78(18), 184203.

40 P. Umari, X. Gonze and A. Pasquarello, Concentration of small ring structures in vitreus silica from a first-principles analysis of the Raman spectrum, Phys. Rev. Lett., 2003, 90(2), 027401.

41 A. G. Revesz and G. E. Walrafen, Structural interpretations for some Raman lines from vitreus silica, J. Non-Cryst. Solids, 1983, 54(3), 323-333.

42 B. Hehlen, Inter-tetrahedra bond angle of permanently densified silicas extracted from their Raman spectra, J. Phys.: Condens. Matter, 2010, 22(2), 025401.

43 L. Svenningsson, Y.-C. Lin, M. Karlsson, A. Martinelli and L. Nordstierna, Molecular Orientation Distribution of Regenerated Cellulose Fibers Investigated with Polarized Raman Spectroscopy, Macromolecules, 2019, 52(10), 3918-3924.

44 D. H. Olson, G. T. Kokotailo, S. L. Lawton and W. M. Meier, Crystal Structure and Structure-Related Properties of ZSM-5, J. Phys. Chem., 1981, 85(15), 2238-2243.

45 To obtain the tensor coefficients the proportionality of the experimentally measured Raman intensity to the laser power, to the number of contributing molecular species under the focal volume, and to the square of the induced dipole were all considered. As an example, to estimate the relation between $\alpha_{x x}$ and $\alpha_{y y}$ the following was used: $I_{x x} / I_{y y}=$ $c \cdot\left(\alpha_{x x}\right)^{2} / c \cdot\left(\alpha_{y y}\right)^{2}$, where the factor $c$ is considered to be a constant throughout measurements performed at different configurations ( $c$ thus includes the number of molecular species illuminated during the measurement and the laser power).

46 Y.-R. Luo. Bond dissociation energies, notendur.hi.is, 2010, pp. 65-98.

47 S. Creci, X. Wang, P.-A. Carlsson and M. Skoglundh, Tuned Acidity for Catalytic Reactions: Synthesis and Characterization of Fe- and Al-MFI Zeotypes, Top. Catal., 2019, 62(7-11), 689-698.

48 P. Brauer, P. L. Ng, O. Situmorang, I. Hitchcock and C. D'Agostino, Effect of Al content on number and location of hydroxyl acid species in zeolites: a DRIFTS quantitative protocol without the need for molar extinction coefficients, RSC Adv., 2017, 7(83), 52604-52613. 